\title{
Fuzzy Supertertiary Interactions within PSD-95 Enable Ligand Binding
}

George L. Hamilton ${ }^{1 *}$, Nabanita Saikia ${ }^{1,4}$, Sujit Basak ${ }^{2}$, Franceine S. Welcome ${ }^{2}$, Fang $\mathrm{Wu}^{2}$, Jakub Kubiak ${ }^{3}$, Changcheng Zhang ${ }^{2}$, Yan $\mathrm{Hao}^{2}$, Claus A.M. Seidel ${ }^{3}$, Feng Ding ${ }^{1 \S}$, Hugo Sanabria ${ }^{1 \S}$, Mark E. Bowen ${ }^{2 \S}$

${ }^{1}$ Department of Physics and Astronomy, Clemson University, Clemson, SC, USA.

${ }^{2}$ Department of Physiology and Biophysics, Stony Brook University, Stony Brook, NY, USA.

${ }^{3}$ Molecular Physical Chemistry, Heinrich Heine University, Dusseldorf, Germany.

${ }^{4}$ Present Address: Department of Chemistry, Navajo Technical University, Chinle, AZ, USA.

* denotes equal contribution.

${ }^{\S}$ To whom correspondence should be addressed: Mark Bowen, mark.bowen@stonybrook.edu; Hugo Sanabria, hsanabr@clemson.edu; Feng Ding, fding@clemson.edu 


\section{ABSTRACT}

The scaffold protein PSD-95 links postsynaptic receptors to sites of presynaptic neurotransmitter release. Flexible linkers between folded domains in PSD-95 enable a dynamic supertertiary structure. Interdomain interactions within the PSG supramodule, formed by PDZ3, SH3 and GuK domains, regulate PSD-95 activity. Here we combined Discrete Molecular Dynamics and single molecule FRET to characterize the PSG supramodule, with time resolution spanning picoseconds to seconds. We used a FRET network to measure distances in full-length PSD-95 and model the conformational ensemble. We found that PDZ3 samples two conformational basins, which we confirmed with disulfide mapping. To understand effects on activity, we measured binding of the synaptic adhesion protein neuroligin. We found that PSD-95 bound neuroligin well at physiological $\mathrm{pH}$ while truncated PDZ3 bound poorly. Our hybrid structural models reveal how the supertertiary context of PDZ3 enables recognition of this critical synaptic ligand. 
The fundamental structural unit of large proteins is the independently folding domain ${ }^{1}$. Most proteins are composed of more than one domain ${ }^{2,3}$. Evolution has shuffled the deck to produce a rich diversity of multidomain proteins. A prototypical example is the Membrane Associated Guanylate Kinases (MAGuKs), which contain an array of folded protein-interaction domains connected in series. MAGuKs are scaffold proteins that link cell surface membrane proteins to their intracellular signaling partners and the cytoskeleton ${ }^{4}$. MAGuK family members control diverse processes ranging from epithelial cell polarity ${ }^{5}$ to synaptic neurotransmitter signaling ${ }^{6}$.

Almost all MAGuKs contain a conserved "PSG" supramodule that links a PDZ domain ${ }^{7}$ an SH3 domain ${ }^{8}$ and guanylate kinase-like (GuK) domain ${ }^{9,10}$ that serves as another protein-binding domain ${ }^{11}$ (Figure 1A). One distinctive feature of the MAGuKs is the insertion of a variable HOOK region within the SH3 domain that disrupts canonical SH3 domain interactions ${ }^{12}$. Different MAGuKs append additional protein-binding domains to this PSG supramodule ${ }^{5}$. The postsynaptic density protein of 95 kilodaltons (PSD-95) contains an N-terminal extension and two tandem PDZ domains attached to its PSG (Figure 1A).

In multidomain proteins, the proximity imparted by interdomain linkers results in high effective concentrations of the folded domains ${ }^{13}$. This can result in specific domain interactions that are too weak to manifest when the domains are not connected ${ }^{14}$. Linkers also allow for different supertertiary arrangements that must be represented by a multiplicity of states ${ }^{15}$. The presence of heterogeneity confounds structural biology methods reliant on ensemble averaging while protein dynamics leads to time averaging even in most single-molecule experiments ${ }^{16}$.

Mapping the conformational landscape of the PSG is important because these supertertiary interactions regulate protein interactions with scaffolding clients ${ }^{17-19}$. Previous SAXS and NMR studies along with computational approaches have proposed binding sites for PDZ3 within SH3-GuK (or a lack thereof) ${ }^{20-22}$. Single-molecule Förster resonance energy transfer (smFRET) studies revealed that PDZ3 was dynamic but had a defined orientation relative to SH3-GuK ${ }^{22}$.

Recently, we combined multiparameter fluorescence measurements with discrete molecular dynamics (DMD) and disulfide mapping to characterize the PDZ tandem from PSD-95 ${ }^{23}$. Here, we applied this approach to the PSG supramodule. Experiments and simulations agreed and revealed two distinct conformational basins for PDZ3; a fuzzy interaction with PDZ3 within a broad interface near the SH3 HOOK insertion and a second discrete binding site in GuK. Both were confirmed with disulfide mapping. Surprisingly, these supertertiary interactions in full-length PSD-95 allowed PDZ3 to interact with the synaptic adhesion protein neuroligin, a known binding partner of PDZ3 ${ }^{24-26}$, while the truncated domain showed weaker binding. Thus, the supertertiary context enhanced the binding activity of PDZ3 towards a critical physiological ligand.

Our integrative approach for dynamic structural biology resolved the structural heterogeneity of the PSG supramodule within full-length PSD-95. Combining simulation with experiments resolved global dynamics and provided residue-level details about supertertiary interactions. This approach moves beyond solving a single structure towards resolving an ensemble of conformers with differing behavior and is applicable to other dynamic multidomain proteins.

\section{RESULTS}

Mapping the Supertertiary Conformational Landscape with Single-Molecule FRET

To experimentally probe the location of PDZ3 within the PSG supramodule (Figure 1A), we used 11 cysteine mutations spanning PDZ3, SH3, and GuK (Table 1). By using these labeling sites in different combinations, we created 12 variants (Figure 1B), which form a FRET network for structural modeling (Figure 1 - figure supplement 1$)^{16}$. Fluorescently labeled proteins were measured with confocal multiparameter fluorescence detection (MFD; for instrument and correction parameters see Supplementary file 1A \&B) and pulsed-interleaved excitation (PIE) that allowed selection of molecules 
with active donor and acceptor fluorophores for sub-ensemble Time-Correlated Single Photon Counting (seTCSPC) (Figure 1C \& Figure 1 - figure supplement 2) ${ }^{27}$. Each variant provides specific information on the same conformational landscape. To capture this shared information, we performed a simultaneous global analysis of all variants ${ }^{28}$. The FRET distances were variant-specific while the number and occupancy of conformational states were set as global fitting parameters. This assumes that the population distribution is the same for all variants, but that each variant senses the underlying conformations differently. Based on fitting statistics, we demonstrate that a two-state model with a small donor-only (or no FRET) population (Supplementary file 1C \&D) is sufficient to fit all data. Thus, PDZ3 samples two limiting states with slight predominance of state B (53.9\%) over state A (46.1\%). The global fit assigns all distances to their respective states (Supplementary file 1E).

For each variant, we plotted the intensity-based FRET efficiency against the average donor fluorescence lifetime $\left(\left\langle\tau_{D(A)}\right\rangle_{f}\right)$ for each molecule (Figure 1D \&

13 Figure 1 - figure supplement 3$)$. We calculated the expected relationship between FRET and $\left\langle\tau_{D(A)}\right\rangle_{f}$ using the assumption of no dynamics, which we plotted as the static FRET-line ${ }^{29}$ (Supplementary file 1F). Molecules undergoing dynamics would fall off this line. The dynamic FRET-lines represent all possible degrees of mixing between the limiting states (Supplementary file $1 G)$. All measured variants exhibited a rightward skew away from the static FRET-line, a hallmark of conformational dynamics (Figure 1D \& Figure 1 - figure supplement 3) ${ }^{29}$.

Variants involving PDZ3 exhibited broad or irregular distributions indicating a heterogeneous conformational ensemble. Variants between PDZ3 and SH3 exhibited higher FRET efficiency, suggesting close proximity. The SH3-GuK domains have restricted interdomain motion ${ }^{12,30}$. Nonetheless, FRET variants spanning SH3-GuK still fell off the static FRET-line. These variants exhibited narrower, more regular FRET distributions relative to PDZ3-labeled variants indicating fast but limited dynamics within SH3-GuK.

Comparison of full-length PSD-95 to the PSG truncation

To probe whether interactions within PSD-95 affect the PSG, we also measured 6 of the FRET variants in a truncated PSG fragment. Measurements using smTIRF with camera detection revealed changes in the timeaveraged FRET distributions for all variants (Figure 2A \& Figure 2 - figure supplement 1). Truncating PSD-95 resulted in broader and more multi-peaked distributions.

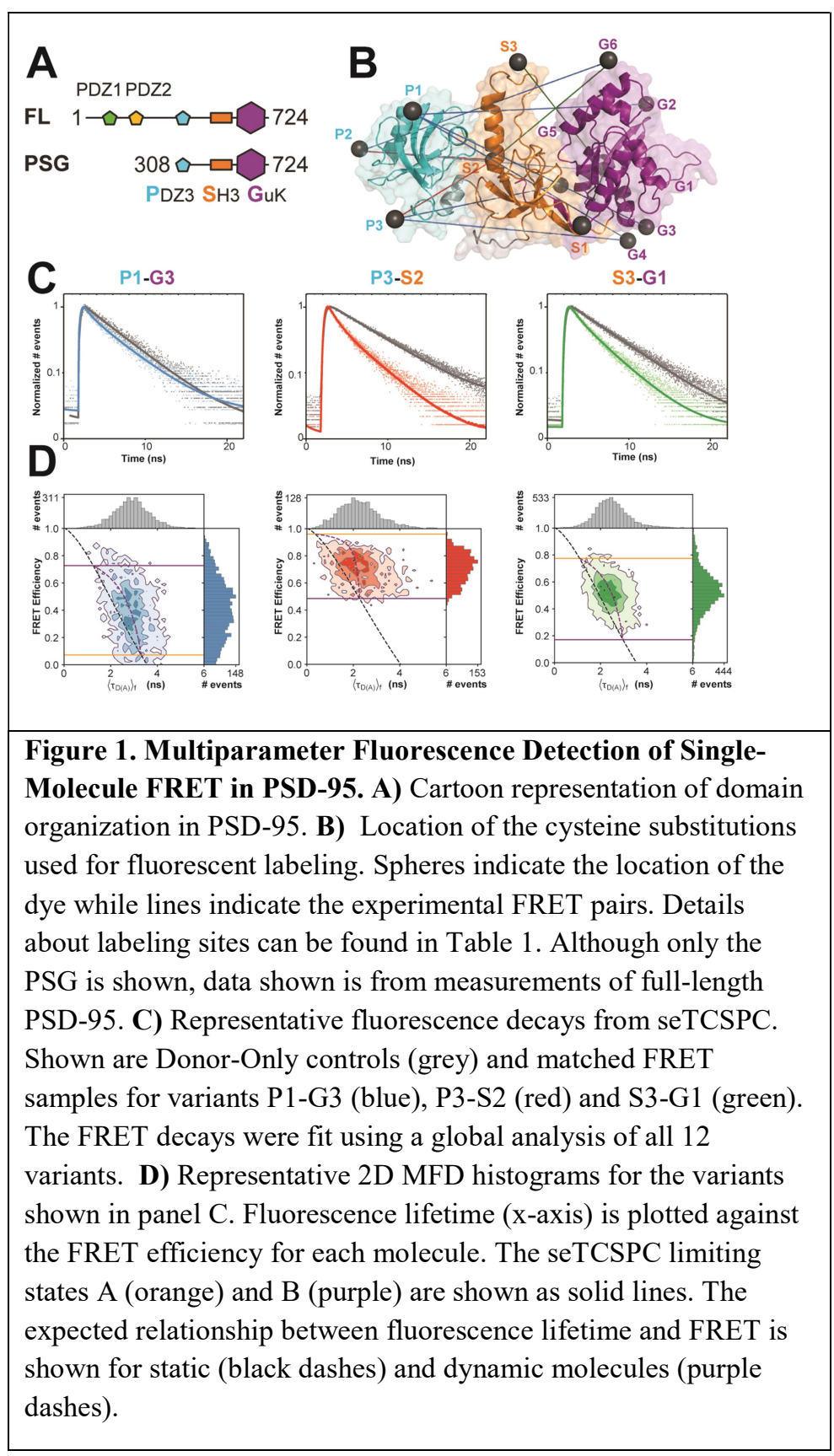
For example, variant P1-G3 (Table 1), splits into lower and higher FRET in the truncation. (Figure 2A). Similarly, variant P3-S2 showed the highest FRET but the distribution spread out to lower FRET when PSD-95 is truncated. Truncation also increased anticorrelated FRET transitions in smTIRF time traces suggesting altered dynamics of PDZ3 (Figure 2 - figure 
supplement 1C). To quantify this, we determined the donor-acceptor cross-correlation amplitude. While the magnitude of the cross-correlation amplitudes depends on FRET efficiency and rate constants, comparison of the same labeling sites in full-length and PSG revealed a uniform increase in FRET transitions when PSD-95 is truncated (Figure 2 - figure supplement 1D).

Measurements with MFD revealed shifts in FRET efficiency and $\left\langle\tau_{D(A)}\right\rangle_{f}$ for most variants (Figure 2 - figure supplement 2). To further analyze the intensity-based FRET efficiencies, we performed Photon Distribution Analysis (PDA) ${ }^{31}$. Our PDA fit model included the two static, limiting states and a dynamic population (Supplementary file 2). Several truncated variants had more molecules in static limiting states, indicating increased dwell time of PDZ3 (Figure 2 - figure supplement 3 ). This suggests the slowest exchange processes were further slowed in truncated variants. However, the predominant state for PDZ3 was always in exchange with faster relaxation rates. PDA additionally found truncation-induced shifts in FRET efficiency similar to smTIRF (Figure 2B). The good agreement between mean FRET efficiencies measured with smTIRF and MFD, representing the long-time averages from both techniques, brings additional confidence in the results (Figure 2C). A global fit of seTCSPC for the PSG recovered two states similar to the full-length protein (Figure 2D \& Figure 2 - figure supplement 4). Truncating PSD-95 shifted the limiting state distances for state A and slightly reduced state B occupancy (48.2\%, Supplementary file 1E).

To resolve fast conformational dynamics, we performed filtered fluorescence correlation spectroscopy (fFCS) ${ }^{32}$. We filtered bursts into subensembles representing the seTCSPC limiting states and analyzed these components using standard correlation algorithms ${ }^{32}$ (Figure 3 - figure supplement 1). Just as FRET efficiency differed between variants, each variant is differently attuned to the same underlying conformational transitions, so data was fit globally to capture the shared information (results in Supplementary file $3 \mathrm{~A} \& \mathrm{~B}$ ). Three decay times were
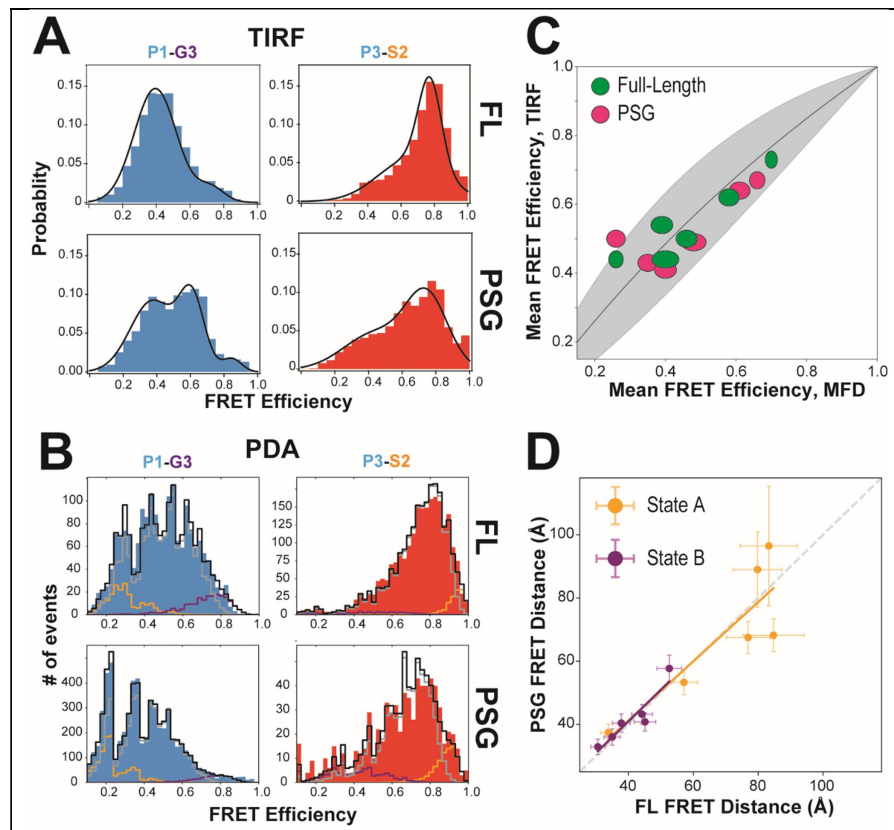

Figure 2. Effect of Truncating PSD-95 on PSG

Supertertiary Structure. A) Representative smTIRF FRET efficiency histograms for full-length PSD-95 (top) and the corresponding truncated PSG fragment (bottom). Shown are variants P1-G3 (blue) and P3-S2 (red). B) Representative PDA plots using a $2 \mathrm{~ms}$ time window for the same variants from panel A using the same coloring. Molecules occupying limiting states $\mathrm{A}$ and $\mathrm{B}$ are highlighted in orange and purple,

respectively. C) Comparison of mean FRET as measured with smTIRF (y-axis) and MFD (x-axis) for full-length (green) and PSG (pink) variants. Ellipse eccentricities represent the relative width of FRET distributions observed by each method. The expected relationship given the different fluorophores used is shown as a line with the shaded region corresponding to Förster Radius uncertainty. Förster Radii used were as used previously ${ }^{22,23}$. The fit to the ideal relationship gave $\chi_{A L L}^{2}=$ 2.13 with $\chi_{F L}^{2}=0.72$ and $\chi_{P S G}^{2}=1.42$. D) Comparison of the seTCSPC limiting-state distances for full-length PSD-95 (yaxis) and the PSG fragment (x-axis). Distances are shown for state A (orange; Slope $=0.94$; Pearson Correlation Coefficient $\left.\left(R_{p}\right)=0.86\right)$ and state B (purple; Slope $=1.0 ; R_{p}=0.93$ ). assigned to local motions $\left(t_{R 1}\right)$ that maintain residue contacts, domain re-orientations $\left(t_{R 2}\right)$ that alter interdomain interaction interfaces, and domain exchange $\left(t_{R 3}\right)$ such as large-scale translational transitions between basins (Figure 3A). PSD-95 variants displayed complex dynamics with components from $\mu$ s to ms. To highlight differences between variants, we plotted the normalized relaxation amplitudes in a matrix representation, which is the dynamics equivalent of a contact map for protein interactions (Figure 3B). This revealed that 7 out of the $10 \mathrm{GuK}$-labeled variants have major (red) or middle (yellow) contributions at $t_{R I}$. The remaining 3 variants are dominated by $t_{R 3}$. The large contribution at $t_{R I}$ for most PDZ3-GuK variants suggests fast local motions within the limiting states, while for P2-G5 and P3-G5 domain exchange dictated 
the dynamics. We also note that for 4 of the remaining 5 PDZ3-GuK variants, the $t_{R 3}$ has middle or major contributions. Moreover, P2-G5, P3-G4, and P3G5 reported middle contributions from domain reorientation.

Summarizing the dynamics observed for the PDZ3GuK variants, fFCS depicts three relaxation times. The major contributions are either $t_{R I}$ suggesting fast domain motions within identified basins (4 out of 7 variants) or $t_{R 3}$ indicating slow jumps between conformational basins ( 3 out of 7 variants). Three variants had their middle contribution at $t_{R 2}$ arising from domain reorientations. Truncated PSG variants exhibited increased heterogeneity in dynamics (Figure 3C) although the major or middle contributions to dynamics appear at $t_{R I}$ or $t_{R 3}$.

\section{Discrete Molecular Dynamics Simulations of the PSG} Core

To map the conformational energy landscape of the truncated PSG supramodule, we performed replica exchange DMD simulations using 18 replicas running at neighboring temperatures with a cumulative total simulation time of $11.9 \mu \mathrm{s}$. To avoid bias, we chose an extended starting conformation with PDZ3 not in contact with SH3-GuK (Figure 4 - figure supplement 1). The probability density function of the radius of gyration $\left(R_{g}\right)$ shows that PDZ3 did not linger in extended conformations, which were rarely sampled (Figure 4A \& Figure 4 - figure supplement 1). Instead, the PDZ3 primarily adopted a docked medium conformation $(\alpha)$ with a mean $R_{g}$ of $27.6 \AA$ along with a more compact conformation $(\beta)$ with mean $R_{g}$ of $23.4 \AA$.

Representative models from these 3 populations (extended, medium, and compact) reveal a diverse ensemble of conformations with PDZ3 sampling both $\mathrm{SH} 3$ and GuK as well as undocked conformations (Figure 4B).

To represent the cumulative association of PDZ3 with $\mathrm{SH} 3$ and $\mathrm{GuK}$, we plotted the distance between centers of mass (COM) for PDZ3 and $\mathrm{SH} 3$ against the distance between COMs for PDZ3 and GuK (Figure 4C). This 2D free energy profile depicts a broad low-energy basin with PDZ3 closer to SH3 (Figure 4C, $\alpha$-basin). This ensemble corresponds to the predominant population in the $\mathrm{R}_{\mathrm{g}}$ distribution. Within the $\alpha$-basin, PDZ3 localized to the HOOK insertion rather than the canonical SH3 domain (Figure 4D). PDZ3 also samples a shallower basin closer to GuK, which is separated by an energy barrier of $\sim 2.0 \mathrm{kcal} / \mathrm{mol}$

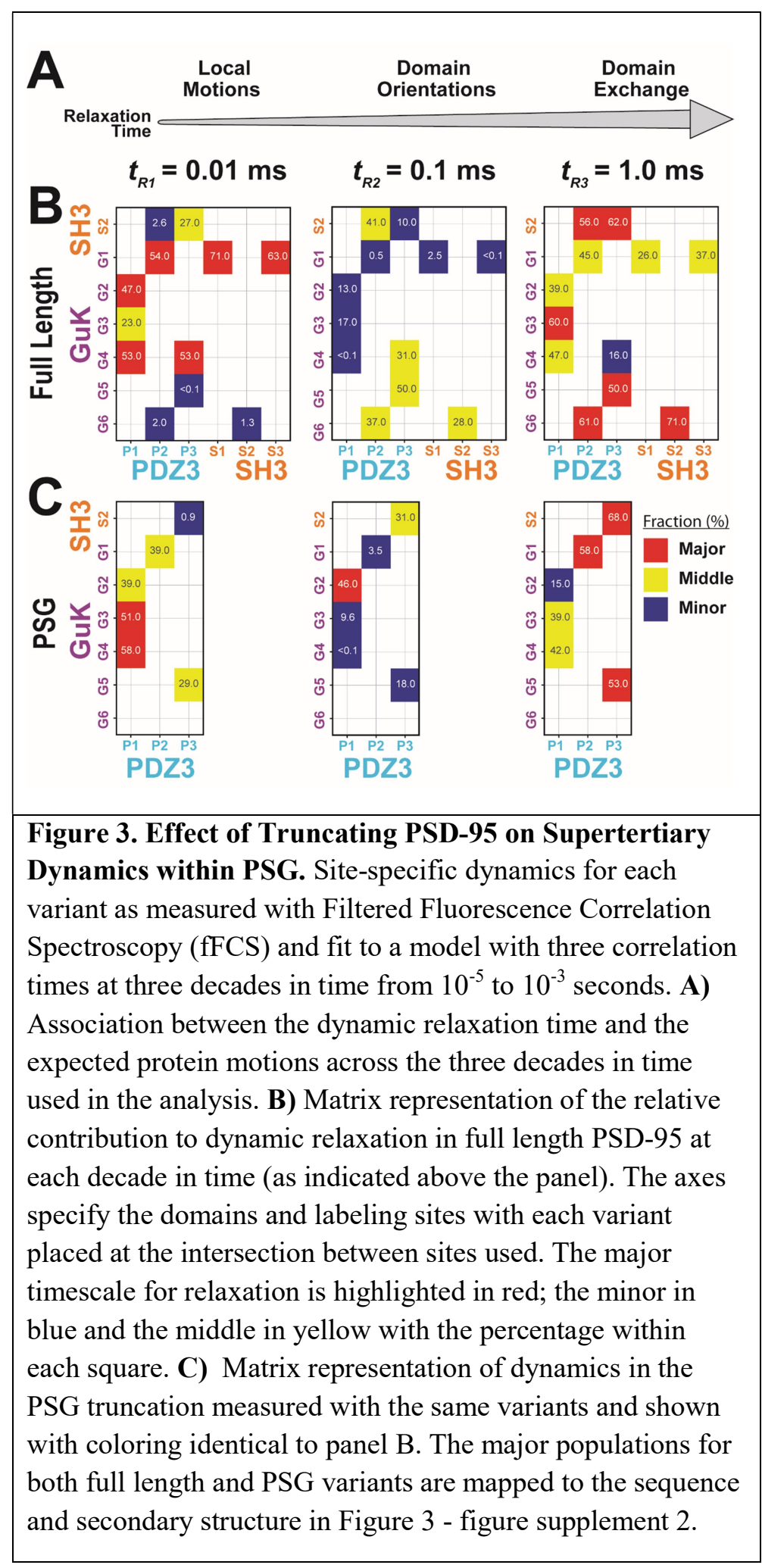


(Figure 4C, $\beta$-basin). This population corresponds to the compact configuration in the $\mathrm{R}_{\mathrm{g}}$ distribution. In addition, PDZ3 samples a range of fully extended conformations with longer distances to both SH3 and GuK (Figure 4C).

Examination of structures within the $\alpha$ basin reveals a broad ensemble of conformations with PDZ3 able to reorient around the HOOK helix and occasionally sample the SH3 RT loop ${ }^{33}$ (Figure 4 - figure supplement 3 ). This suggests a fuzzy and dynamic interaction. Examination of $\alpha$-basin pairwise contacts reveals that basic residues within HOOK interact with negatively charged residues in PDZ3 and are further stabilized by surface-exposed aromatic and hydrophobic residues (Figure 4E). Negatively charged residues in $\alpha 3$ and the $\beta 2-\beta 3$ loop of PDZ3 keep the peptidebinding face oriented towards SH3. Steric clashes would preclude PDZ3 from ligands binding while in some conformations within the $\alpha$-basin (Figure 4 - figure supplement 3). PDZ3 interacts directly with SH3 rather than having this interaction mediated by the linker as had been proposed ${ }^{20}$. Instead, the PDZ3-SH3 linker interacts with the $\mathrm{SH} 3$ domain, which helps retain PDZ3 in the $\alpha$ basin and prevents more extended conformations.

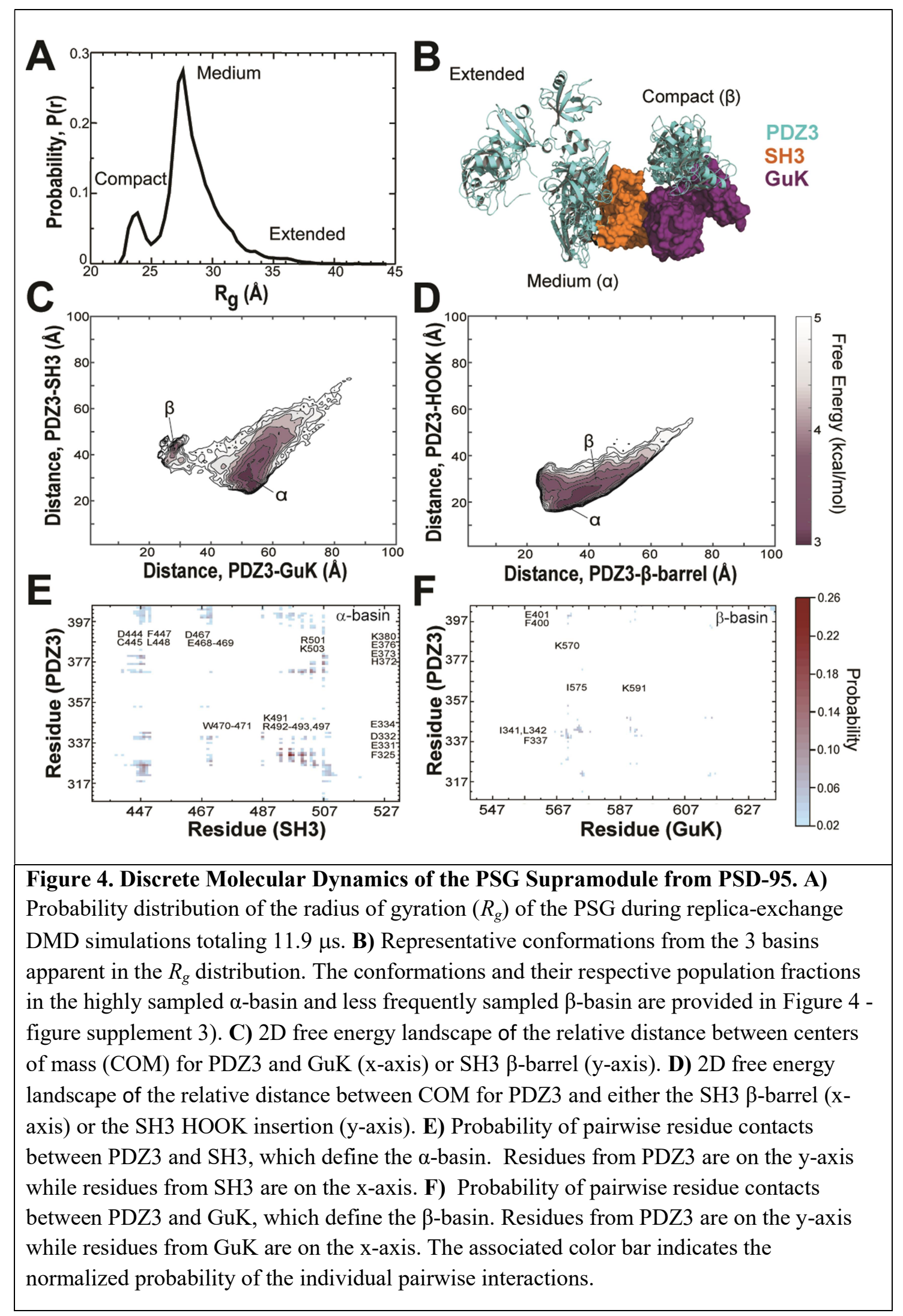


Hydrophobic and electrostatic interactions between the PDZ3-proximal linker (F400, E401, K403 and I404) and SH3 are among the top 50 pairwise residue contacts.

Examination of the $\beta$-basin ensemble revealed a more well-defined interaction with PDZ3 binding near the interface of the nucleoside monophosphate (NMP) binding and CORE subdomains of GuK (Figure 4B). The interaction does not

\begin{abstract}
occlude the canonical peptide binding sites in GuK or PDZ3 (Figure 4 - figure supplement 3 ). Analysis of the $\beta$-basin
\end{abstract} pairwise contacts revealed a well-defined binding site stabilized by hydrophobic and hydrogen bonding interactions between uncharged polar residues (Figure 4F) unlike the highly charged interface in the $\alpha$-basin. Interestingly, the PDZ3SH3 linker also makes significant pairwise contacts with GuK in this basin. Unexpectedly, the HOOK insertion formed an extended $\alpha$-helix between residues 491 and 508 in DMD simulations. This is longer than observed in crystal structures ${ }^{12,30}$ (Figure 4 - figure supplement 4). Interestingly, PDZ3 interactions appear to stabilize the helical extension as is visible in the representative basin models (Figure 4 - figure supplement 3 ).

\section{Disulfide mapping the interdomain interfaces}

To confirm the PDZ3 interactions with SH3 and GuK, we engineered cysteine residues based on the contact frequency maps and measured the extent and rate of disulfide bond (DS) formation (Figure 5). DS formation depends on distance and orientation between cysteines but also contact frequency for dynamic interactions. Thus, the kinetics of bond formation report on structural proximity ${ }^{34}$. We made peripheral cysteine substitutions so as to not disrupt the primary contacts, which are shown using the structure with the lowest root mean square deviation (RMSD) to each basin ensemble. The predominant interface in the $\alpha$-basin was PDZ3 binding to the HOOK. We probed this basin with the residue pair N326C-A504C, which are only $5.6 \AA$ apart in the $\alpha$-basin representative (Figure 5A). The predominant interface in the $\beta$-basin had PDZ3 binding to GuK. We probed this basin with the residue pair G344C-N633C, which are $5.7 \AA$ apart in the $\beta$-basin representative (Figure 5B). 
Increased electrophoretic mobility indicated that DS formation was occurring for all samples (Figure 5 - figure supplement 1). The data were fit to an exponential function to determine the rate and final extent of DS formation (Figure 5D). The $\alpha$-basin variant showed slightly more DS formation than the $\beta$-basin variant in full-length PSD-95 but the rates of DS formation were similar (Figure 5E \& F). To probe the effects of truncation, we measured DS formation in the PSG truncation. Interestingly, the truncation had opposite effects on the kinetics of DS formation for the two variants. The rate of DS formation for the $\alpha$-basin variant increased by $\sim 30 \%$ while rate of DS formation for the $\beta$-basin variant decreased by six-fold.

We also screened the DMD simulations for FRET variants capable of DS formation, which predicted that variant P3$\mathrm{S} 2$ occasionally sampled close distances in the $\alpha$-basin (Figure 5C). As predicted, variant P3-S2 formed DS albeit to a lesser extent and much more slowly than either designed variant. The extent of DS formation was slightly higher in the truncation, but the rates were similar. Thus, DS formation for all three variants was higher in the truncation. Our DS analysis confirms that the contact interfaces from DMD are sampled in full-length and truncated PSD-95. We also observe significant kinetic differences when PSD-95 is truncated in agreement with FRET studies.

\section{Structural Modeling with Experimental FRET Restraints}

To describe the conformational ensemble associated with each limiting state from seTCSPC, we used the FRET distances as restraints in structural modeling. We simulated the accessible volume (AV) for both dyes at each labeling site

${ }^{35}$ (Supplementary file 4A), which we used as distance loci for rigid body docking. For each state, we generated 33000 conformations, which were each scored for agreement between the AV model distances and the FRET distances. For state A, the best-fit models showed some divergence with PDZ3 near the HOOK insertion but also extended without interdomain contacts (Figure 6A). The best-fit models for state B are more tightly clustered and position PDZ3 near the NMP subdomain of GuK (Figure 6B).

To independently corroborate the docking models, we calculated the AV for all snapshots structures from the DMD trajectory. For each structure, we plotted the inter-AV distance $\left(\left\langle R_{D A}\right\rangle_{A V}\right)$ against the distance between the COM of labeled domains (Figure 6 - figure supplement 1 ). To denote the limiting states, we overlay orange and purple vertical lines for state A and B respectively. The limiting states A and B generally fall within the associated $\alpha$ - and $\beta$-basins. However, it was also clear that for some variants, (e.g. P2-G6) the vertical line for state B agrees with both DMD basins. Similarly, the PDZ3-SH3 variants may not fully capture the underlying population distribution. This apparent discrepancy rises from what we call FRET degeneracy in which a single distance captures an underlying heterogenous distribution. To resolve the FRET degeneracy, we introduce the FRET Network Robustness (FNR) analysis (Figure 6 - figure supplement 2). We systematically refit the seTCSPC data using different numbers and combinations of variants from the FRET network. We randomly selected sub-samples with as few as 5 variants while including each variant in at least 3 subsets. The distance deviation increased with fewer variants, but the FNR distributions remained centered on the distance from the full global fit (Figure 6 - figure supplement 2). When more than 7 variants were used, the FNR deviation was less than $10 \%$ regardless of which variants were included in the subset. The standard deviation of the FNR distributions (along with AV simulations) captures the heterogeneity introduced by FRET degeneracy, which can be used to impose new bounds on structural heterogeneity (Supplementary file 4B).

Using these bounds, we screened the 20871 snapshot structures from DMD simulations to classify all models that fall within these new bounds for the $\alpha$ - and $\beta$-basins. Displaying the PDZ3 COM for each compatible model as a cyan sphere emphasizes the fuzziness of the basins as captured by the underlying heterogeneous FRET distributions from each variant. The broad ensemble for the $\alpha$-basin is quantified by the variance of the FNR distributions (Figure 6 - figure supplement 2) but arises from the fuzziness of interdomain interactions between PDZ3 and SH3 (Figure 6C). In contrast, the $\beta$-basin is not fuzzy (Figure 6D). For comparison, we overlay the COM of screened DMD snapshots that fall within the 95\% confidence interval excluding contributions from FRET degeneracy (Figure 6C and 6D; grey spheres). 
We used this

2 information to

3 construct a

4 conformational

5 landscape

6 consistent with

7 DMD and FRET

8 (Figure 6E). We

9 performed

principal

component analysis and projected the basins along the first two principal components (PC1 and PC2) using the $\mathrm{COM}$ distances and the simulated $\left\langle R_{D A}\right\rangle$ between PDZ3 and SH3-GuK, which were rescaled such that the integrated volume of each basin was equivalent to its population fraction from seTCSPC

(Figure 6-

figure

supplement 2).
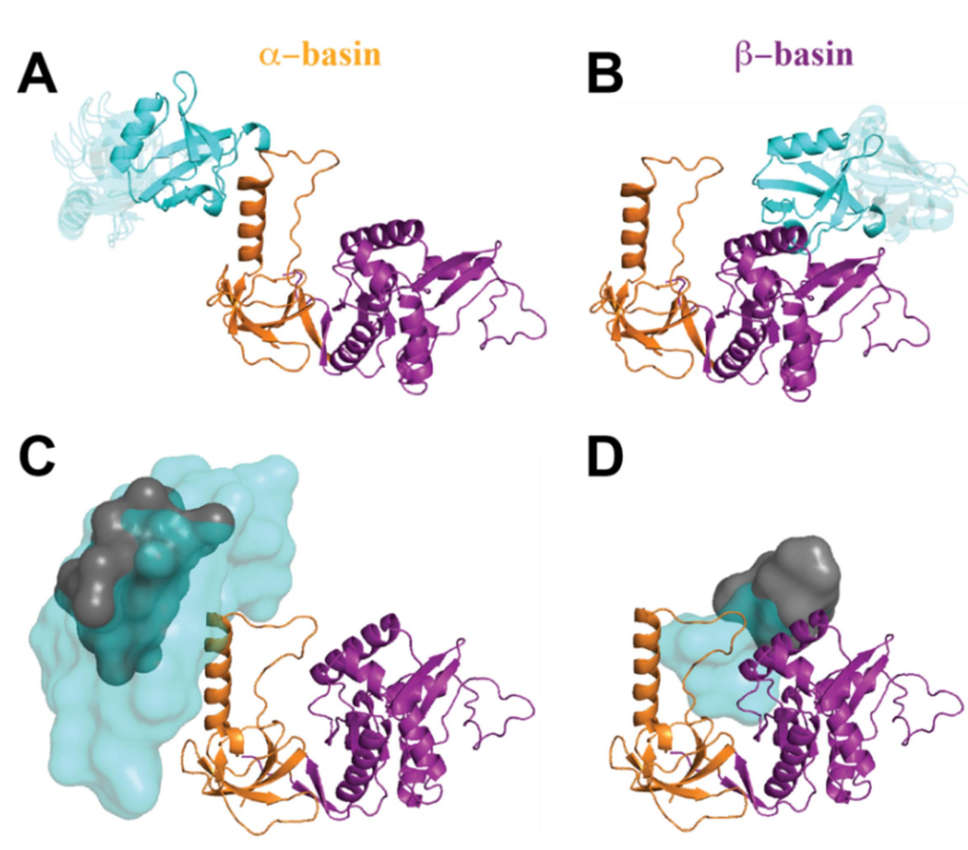

D

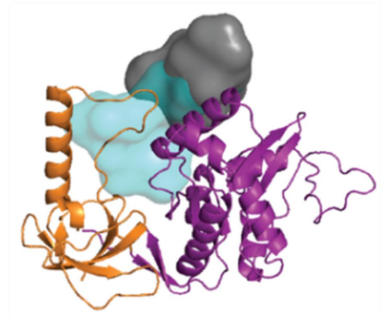

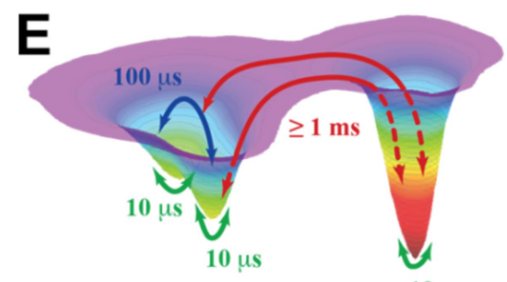

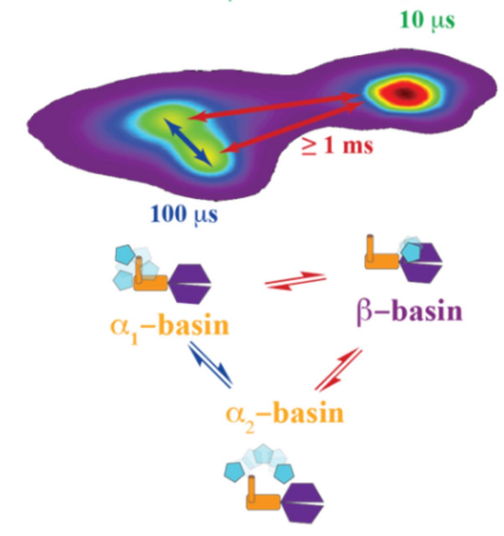

\section{Figure 6. Modeling the Supertertiary Structural Ensemble with Experimental FRET}

Restraints. A-B) Cartoon representations of the best fit models from rigid body docking of PDZ3 based on the FRET distances from seTCSPC (PDZ3, cyan; SH3; orange; GuK, purple). A) Best fit model for full-length PSD-95 in limiting state A. (B) Best fit model for limiting state B. C-D) Heterogeneity of the conformational state ensembles based on classification of structures from DMD trajectories into state A (C) or state B (D). The grey surfaces represent $95 \%$ confidence intervals for localization of the PDZ3 center of mass based on screening with limiting state distances from all 12 full-length variants. Confidence intervals are based on the F-test for the ratio of $\chi_{r}^{2}$ for all docking structures relative to the $\chi_{\mathrm{r}}^{2}$ of the top-ranked structure with 9 free parameters (number of distances used for docking of PDZ3). The grey surfaces represent only the uncertainty in distances from the global fit but do not capture the full heterogeneity of each basin. The cyan surfaces represent conformational space accessible to the PDZ3 center of mass from screening DMD with thresholds from FNR reanalysis of seTCSPC data ( Figure 6 - figure supplement $1 \& 2$, Supplementary file 4B). E) Energy landscape for the conformational ensemble of the PSG supramodule within PSD-95. Transitions and their associated timescales taken from fFCS indicated by colored arrows. The fastest transitions are associated with local motions, which were not resolved by FRET but are inferred from DMD simulations and fFCS. The energy landscape surface was generated by rescaling the surface that resulted from principal component analysis ( Figure 6 - figure supplement 3 ) such that the integrated volumes of basins $\alpha$ and $\beta$ matched the population fractions for states $\mathrm{A}$ and $\mathrm{B}$, respectively, from TCSPC analysis. Basins $\alpha_{1}$ and $\alpha_{2}$ correspond to the two sub-basins separated by a small shoulder along principal component 2 (Figure 6 - figure supplement 3 ). the $\alpha$ - and $\beta$ -
basins mostly by interdomain displacement, while PC2 describes the $\alpha$-basin FRET degeneracy due to domain reorientations within a single basin. Hence, we expand the energy landscape to include degenerate $\alpha_{1}$ - and $\alpha_{2}$ basins and link the fFCS relaxation rates directly to the barrier heights for transitions between basins.

\section{Effect of PDZ3 Native Context on Interactions with Neuroligin}


Our PSG models include conformations that could impact ligand binding to PDZ3. To test this, we examined the interaction with neuroligin 1a, a key synaptic adhesion protein that interacts with PDZ3 (Figure 7A) ${ }^{24,25}$. To compare binding between the truncated PDZ3 and full-length PSD-95, we used a 10 residue C-terminal neuroligin peptide (NL10), which bound truncated PDZ3 with an equilibrium dissociation constant $\left(K_{D}\right)$ of $10 \mu \mathrm{M}^{26}$. We N-terminally-labeled the peptide with fluorescein and measured the fluorescence anisotropy.

We obtained a $K_{D}$ of $25 \pm 4$ $\mu \mathrm{M}$ at $\mathrm{pH} 6$, which is in good agreement given our higher ionic strength. Surprisingly, we were unable to reach saturation when we repeated this measurement at pH 7.4 indicating that the $K_{D}$ increased to over $200 \mu \mathrm{M}$ (Figure 7B). Thus, neuroligin binding showed strong $\mathrm{pH}$ dependence and bound to PDZ3 poorly at physiological $\mathrm{pH}$. In contrast to truncated PDZ3, full length PSD95 gave a $K_{D}$ of $15 \pm 1 \mu \mathrm{M}$ at $\mathrm{pH}$ 6 and $\mathrm{pH} 7.4$ suggesting the NL10 interaction is enhanced by the supertertiary environment of PDZ3. Next, we examined neuroligin binding to the PSG truncation. We measured a $K_{D}$ of $39 \pm 1 \mu \mathrm{M}$ at $\mathrm{pH} 6$ suggesting that binding affinity is somewhat reduced in the PSG relative to full length or even the PDZ3 truncation. The PSG showed slightly higher binding affinity at $\mathrm{pH} 7.4$ with a $\mathrm{K}_{\mathrm{D}}$ of $31 \pm 2 \mu \mathrm{M}$ in stark contrast to the reduced binding affinity of the truncated PDZ3. Thus, effects of truncation on supertertiary structure and dynamics impact NL10 binding.

\section{To understand this}

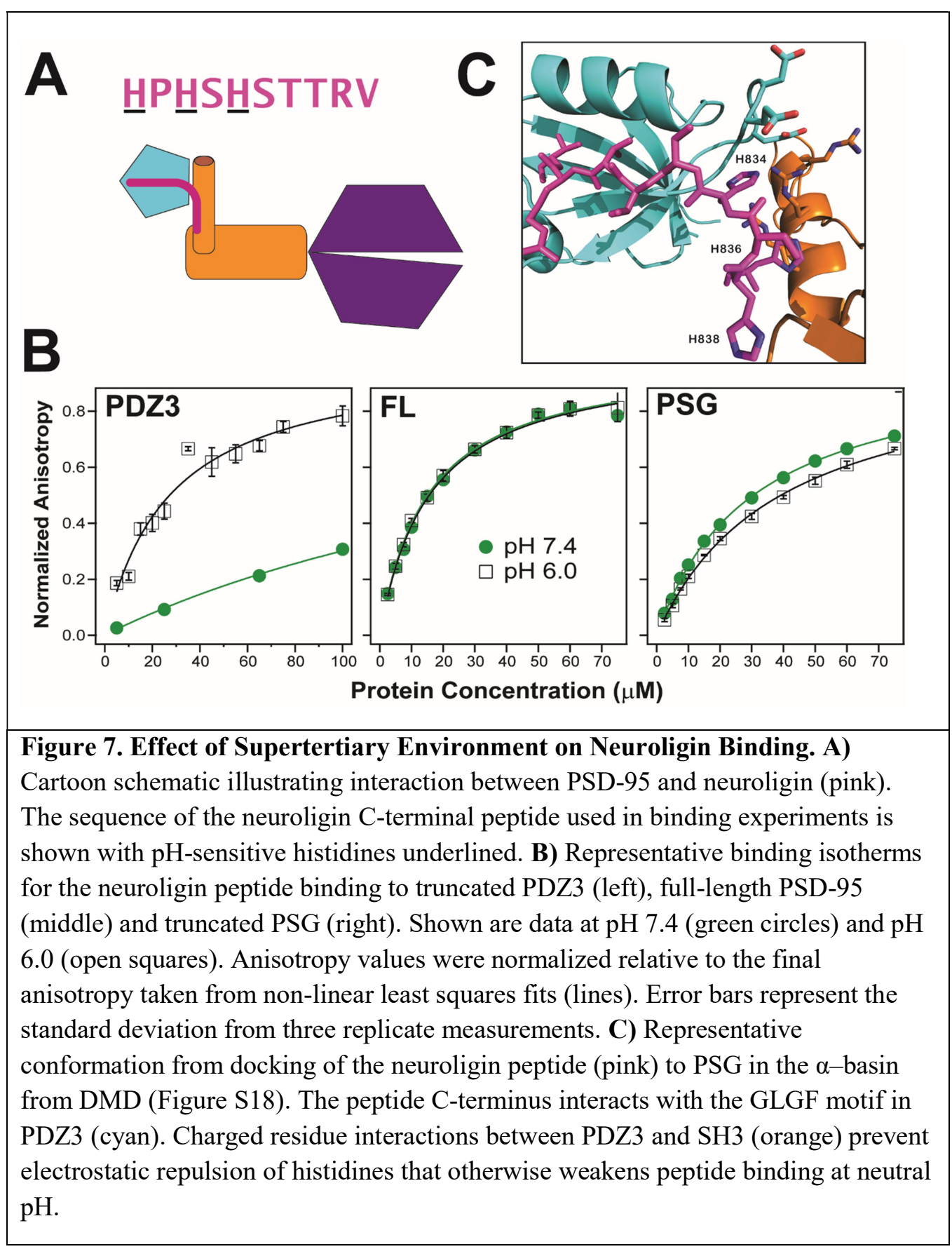
phenomenon, we performed docking of NL10 to truncated PDZ3 at pH 6. We identified electrostatic interactions between protonated histidines in NL10 and acidic residues in the PDZ3 $\beta 2-\beta 3$ loop (Figure 7 - figure supplement 1). At pH 7.4, unprotonated histidines are incapable of interacting, which explains the $\mathrm{pH}$ sensitivity. The resulting desolvation penalty of the unpaired acidic residues would reduce the binding affinity. Some $\alpha$-basin conformations block peptide binding due to steric clashes. However, docking of NL10 to $\alpha$-basin structures identified multiple PSG conformations where the acidic residues in PDZ3 interacted with basic residues in HOOK without steric clashes for NL10 (Figure 7C). Salt-bridges have 
a smaller desolvation penalty than unsatisfied charges, which explains why the peptide binds full-length PSD-95 (and PSG) better than truncated PDZ3 at pH7.4. Thus, the supertertiary context of PDZ3 enables recognition of a critical

3 physiological ligand.

\section{DISCUSSON}

PSD-95 is a scaffold protein at excitatory synapses that forms a crucial link between neurotransmitter release and soluble enzymes. Proteomic analysis of PSD-95 complexes purified from mouse brain identified 301 different proteins ${ }^{36}$. In many cases, the binding partners are larger than the scaffolding domains with which they interact. Kinetic analysis showed that higher-order interactions, between proteins bound to PSD-95, plays a role in scaffolding activity ${ }^{37}$. The assembly of multi-protein complexes poses steric challenges. Thus, the dynamic supertertiary structure must play a role in accommodating multiple partners.

Here we used a combination of experimental and computational methods to describe the supertertiary structure and dynamics of the conserved PSG supramodule within full-length PSD-95. Multiparameter fluorescence analysis revealed a complex and dynamic conformational landscape. DMD and modeling based on FRET distances were in excellent agreement on the location of PDZ3 within two non-overlapping basins. Both DMD and FRET agree that the $\alpha$-basin was degenerate due to the fuzzy interface allowing PDZ3 reorientation. The fFCS was crucial for assigning dynamic timescales to the conformational transitions, which we summarized as an energy landscape (Figure 6E). Within each basin, each domain undergoes rapid local motions. Reorientation of PDZ3 and sampling of extended states is slower while basin exchange take place on timescales approaching seconds as captured by smTIRF.

Our models were in excellent agreement with SAXS and NMR experiments, which identified multiple conformations with PDZ3 predominantly localized near the HOOK insertion and suggested involvement of residues around the PDZ3 peptide binding pocket ${ }^{20}$. However, Rosetta modeling suggested the PDZ3-SH3 linker bridged the interaction while we found the PDZ3-SH3 interaction was direct. The linker interacts with SH3 and GuK to help retain PDZ3. This linker is almost 100\% conserved in PSD-95 homologues from humans to drosophila, which would be unusual unless involved in protein interactions since disordered linkers usually show reduced sequence conservation ${ }^{38}$. Mutations in the PDZ3-SH3 linker could explain the reduced interactions seen in NMR on re-engineered PSG constructs ${ }^{39}$. Neither basin relied on canonical ligand binding modes for the primary interaction as suggested by comparative patch analysis ${ }^{21}$.

The crystal structure of the PSG supramodule from ZO-1, a MAGuK protein found at intercellular tight junctions, revealed a lack of interdomain interactions ${ }^{40}$. The PDZ3-SH3 linker in ZO-1 is much shorter than PSD-95, which may prevent access to these binding sites. The ZO-1 HOOK insertion also lacks sequence similarity to PSD-95 ${ }^{40}$. In contrast, the basic residues in HOOK and acidic residues in PDZ3 are conserved among synaptic MAGuK homologs PSD-93, SAP97, and SAP102 as are most pairwise contacts in the $\beta$-basin (e.g., F339-Q594, L342-Q603). This explains why the supertertiary landscape is conserved in these homologues as suggested by previous smTIRF measurements ${ }^{22}$.

The importance of supertertiary interactions on scaffolding activity is emphasized by the enhanced binding of neuroligin to full-length PSD-95. Other PDZ3 ligands (e.g., CRIPT, stargazin and synGAP) contain lysines and arginines near the canonical PDZ motif while neuroligin contains histidines, which explains why NL10 has pH sensitivity. In the fuzzy $\alpha$-basin, interactions with positive charges in the HOOK satisfy negatively charged residues in PDZ3, which facilitates uncharged peptide binding. Additionally, fuzziness increases the favorability of the $\alpha$-basin by allowing PDZ3 to reorient to satisfy multiple interactions. These supertertiary interactions also affect other ligands. Measurements of CRIPT binding to PDZ3 suggested that PDZ3 adopted two interconverting conformational states in the PSG with different kinetics but the same equilibrium affinity ${ }^{41}$. Thus, the supertertiary context of PDZ3 is necessary to overcome the repulsive interactions that prevent neuroligin binding to the truncated domain. 


\section{Protein expression and purification}

The full-length PSD-95 from Rattus norvegicus, the PSG truncation (residues 303-274) and truncated PDZ3 (303-415) were expressed in the Rosetta 2 strain of E. coli (EMD Millipore) and purified by a combination of Ni-affinity, ion exchange and size exclusion chromatography as previously described ${ }^{14}$. For each construct, we prepared a series of labeling variants using standard site directed mutagenesis and confirmed with DNA sequencing (Table 1). For smTIRF, proteins were labeled Alexa Fluor $555 \mathrm{C}_{2}$ maleimide and Alexa Fluor $647 \mathrm{C}_{2}$ maleimide at an equimolar ratio. For MFD, proteins were first labeled with a 1:2 ratio of Alexa $488 \mathrm{C}_{5}$ maleimide for 1 hour at $4^{\circ} \mathrm{C}$ followed by addition of a 5:1 molar ratio of Alexa Fluor $647 \mathrm{C}_{2}$ maleimide, which was reacted overnight at $4^{\circ} \mathrm{C}$. Unconjugated dye was removed by

\begin{tabular}{|c|c|c|}
\hline Position & Mutation & \multirow{13}{*}{$\begin{array}{l}\text { Table 1. Labeling sites } \\
\text { used for single- } \\
\text { molecule FRET } \\
\text { measurements. The } \\
\text { labeling position is } \\
\text { identified first by a } \\
\text { single letter indicating } \\
\text { the domain (P for } \\
\text { PDZ3, S for SH3 and G } \\
\text { for GuK) followed by } \\
\text { its sequential order in } \\
\text { the primary sequence. }\end{array}$} \\
\hline P1 & R313C & \\
\hline P2 & Q374C & \\
\hline P3 & S398C & \\
\hline S1 & $\mathrm{H} 477 \mathrm{C}$ & \\
\hline S2 & R492C & \\
\hline S3 & S510C & \\
\hline G1 & K591C & \\
\hline G2 & S606C & \\
\hline G3 & E618C & \\
\hline G4 & E621C & \\
\hline G5 & $\mathrm{A} 640 \mathrm{C}$ & \\
\hline G6 & R671C & \\
\hline
\end{tabular}
desalting with Sephadex G50 (GE Healthcare) followed by dialysis.

\section{Single-Molecule Total Internal Reflection Fluorescence (TIRF)}

Fluorescently labeled PSD-95 was encapsulated in $100 \mathrm{~nm}$ liposomes supplemented with $0.1 \%$ biotinylated phosphoethanolamine (Avanti Polar Lipids, Alabaster, AL). Unencapsulated protein was removed by desalting on Sepharose CL-4B (GE Healthcare). Liposomes were attached via streptavidin to a quartz slide passivated with biotinylated-BSA. FRET data was collected at 10 frames/second using an Andor iXon EMCCD camera (Andor Technologies, Belfast, UK). All smFRET measurement were performed in $50 \mathrm{~mm}$ Tris $150 \mathrm{mM} \mathrm{NaCl} \mathrm{pH} 7.5$ supplemented with $5 \mathrm{mM}$ cycooctatetraene, $0.5 \% \mathrm{w} / \mathrm{v}$ glucose, $7.5 \mathrm{units} / \mathrm{mL}$ glucose oxidase and 100 units $/ \mathrm{mL}$ catalase. Alternating illumination using 640 and $532 \mathrm{~nm}$ lasers allowed for the identification of molecules containing one donor and one acceptor. Microscopy data was analyzed using home written scripts in MATLAB to correlate donor and acceptor images, extract single-molecule intensity time traces from the pixel intensity and calculate FRET efficiency with corrections for background and crosstalk. Automated per molecule gamma normalization based upon acceptor photobleaching events was used to correct for distortions in the measured intensities between the donor and acceptor channels $^{42}$.

\section{Multiparameter Fluorescence Detection}

Two experimental setups were used for confocal measurements to obtain MFD data (Supplementary file 1A \& B). For the Clemson University setup, freely diffusing molecules were excited as they passed through the focal volume of a $60 \mathrm{X}$, 1.2 NA collar (0.17) corrected Olympus objective with diode lasers at $485 \mathrm{~nm}$ and $640 \mathrm{~nm}$ (PicoQuant, Germany) operating at $40 \mathrm{MHz}$ with $25 \mathrm{~ns}$ interleaved time. The power at the objective was $80 \mu \mathrm{W}$ at $485 \mathrm{~nm}$ and $32 \mu \mathrm{W}$ at $640 \mathrm{~nm}$. Emitted photons were collected through the same objective and spatially filtered through a $70 \mu \mathrm{m}$ pinhole to limit the effective confocal detection volume. For the setup located at Heinrich Heine University (HHU), molecules were excited with diode lasers at $485 \mathrm{~nm}$ and $640 \mathrm{~nm}$ (PicoQuant, Germany) operating at $64 \mathrm{MHz}$ (15.6 ns interleaved time). The power at the objective was set to $60 \mu \mathrm{W}$ at $485 \mathrm{~nm}$ and $10 \mu \mathrm{W}$ at $640 \mathrm{~nm}$. The location at which each sample was measured is indicated in Supplementary file 1A.

Emission was separated into parallel and perpendicular polarization components at two different spectral windows using band pass filters, ET525/50 and ET720/150, for donor and acceptor, respectively (Chroma Technology Co.). In total, four photon-detectors are used at the Clemson setup - two for donor (PMA Hybrid model 40 PicoQuant, Germany) and two for acceptor (PMA Hybrid model 50, PicoQuant, Germany). To ensure temporal data registration of the 4 synchronized input channels, we used a HydraHarp 400 TCSPC module (PicoQuant, Germany) in Time-Tagged Time- 
1 Resolved mode. For the HHU setup, eight detection channels are used-four for green ( $\tau$-SPAD, PicoQuant, Germany)

2 and four for red APD SPCM-AQR-14, Perkin Elmer, Germany) data registration synchronization was achieved with

3 HydraHarp 400 TCSPC module (PicoQuant, Germany) in Time-Tagged Time-Resolved mode.

Both setups utilized Pulsed Interleaved Excitation (PIE) to alternately excite donor and acceptor fluorophores directly ${ }^{43}$. Emission was separated into parallel and perpendicular polarization components at two different spectral windows using band pass filters as described in supplementary methods. Labeled samples were diluted in $50 \mathrm{mM}$ sodium phosphate, $\mathrm{pH} 7.5,150 \mathrm{mM} \mathrm{NaCl}, 40 \mu \mathrm{M}$ TROLOX, which had been charcoal filtered to remove residual impurities. We used $\mathrm{pM}$ concentrations such that we observed $\sim 1$ molecule per second in the confocal volume. Samples were measured in NUNC chambers (Lab-Tek, Thermo Scientific, Germany) that were pre-coated with a solution of $0.01 \%$ Tween 20 (Thermo Scientific) in water for $30 \mathrm{~min}$ to minimize surface adsorption. We obtained the instrument response function (IRF) using water. Protein-free buffer was used for background subtraction. Calibration experiments and data collection were as previously reported ${ }^{44}$. Burst selection was performed using all-photon, inter-photon arrival time traces to identify single molecules. Burst selection criteria were set such that each burst contained a minimum of 40 photons summed amongst all detection channels, with an inter-photon arrival time cutoff set to the mean minus one standard deviation, calculated across the entire time trace. The donor fluorescence lifetime and the intensity-based FRET efficiency were calculated for each burst using a maximum-likelihood estimation algorithm ${ }^{23,45}$. To ensure both fluorophores were present in each select burst used in MFD histograms, we used cutoff values for 1) the difference between observed burst duration in green and red channels under direct excitation of the corresponding fluorophores $\left(\left|\mathrm{T}_{\mathrm{GG}}-\mathrm{T}_{\mathrm{RR}}\right|<1 \mathrm{~ms}\right)$ and 2) the observed FRET stoichiometry $\left(0.3<\mathrm{S}_{\mathrm{PIE}}<0.7\right)^{23}$.

\section{Subensemble Time-Correlated Single-Photon Counting (seTCSPC) Analysis}

Analysis of donor fluorescence decays in conditions for FRET to determine limiting states was performed on photon data corresponding to bursts shown in Figure 1 - figure supplement 3. These fits and raw data are shown in Figure $1-$ figure supplement 2 and Figure 2 - figure supplement 2 for full-length and truncated PSG core, respectively. Data for fits was generated by loading burstwise data and IRF data corresponding to the green detection channels into the FitMachine analysis software using 4096 TAC bins and saving the resulting fluorescence decay and IRF histograms. Fitting was performed in the ChiSurf analysis software, which allows users to set individual parameters to be shared globally and optimized amongst subsets of curves. Fit models were multi-exponential decays described by:

$$
f_{D(A)}(t)=\sum_{i=1}^{2} x_{i} e^{-t / \tau_{D, i}}+x_{D(0)} e^{-t / \tau_{D(0)}}
$$

where $x_{i}$ and $x_{D(0)}=1-\sum_{i=1}^{2} x_{i}$ are species fractions of exponential decay terms for FRET-sensitized and DO/no-FRET emission, and $\tau_{D, i}$ and $\tau_{D(0)}$ are the corresponding donor fluorescence decay lifetimes. The instrument response function (IRF) is deconvolved and decays are related to states with underlying Gaussian-distributed interdye distances as described 23,46. Prior to global fitting, donor-only (DO) fluorescence lifetimes and fractions were determined by fitting each variant with the corresponding DO curves. These DO curves are shown in Figure 1 - figure supplement 2 and Figure 2 - figure supplement 2, and the DO lifetimes and fractions are provided in Supplementary file 1D. Global fits for the full-length variants were performed by loading all decay data simultaneously along with the corresponding IRF data. DO fluorescence lifetimes, DO fractions, and instrumental parameters were fixed for each sample based on DO fits, and two distances corresponding to donor fluorescence lifetimes and fractions were used for each FRET variant, with population fractions set as global parameters and distances optimized individually. The fit parameters for this analysis are listed in Supplementary file $1 \mathrm{D}$. Where $\tau_{D, x i}$ and $\tau_{D, f i}$ are used, $x$ indicates the species mean fluorescence lifetime used in seTCSPC fits and $f$ indicates the fluorescence-weighted average lifetime for the state used in computing FRET-lines for MFD histograms. Conversion to distances was performed as we have done previously, using a standard Förster radius of $52 \AA$ for the Alexa 488 and Alexa 647 dye pair (2). The Förster radius was corrected individually for each sample based on the corresponding mean dye orientation factor, $\left\langle\kappa^{2}\right\rangle$, given in Supplementary file 1D. The expression for $\left\langle\kappa^{2}\right\rangle$ and relation to the Förster radius has been detailed previously ${ }^{47}$. The values of $\left\langle\kappa^{2}\right\rangle$ were estimated using the Kappa2- 
Distribution tool in the Chisurf software package. The values of steady-state anisotropy used for these estimations $\left(\mathrm{r}_{\mathrm{D} D \mathrm{D}, \infty}\right.$, $\mathrm{r}_{\mathrm{A} \mid \mathrm{A}, \infty}$ for donor and acceptor under direct excitation, respectively) were calculated from MFD histograms and are provided in Supplementary file 1D. Interdye distances from the seTCSPC fitting can be found in Supplementary file 1E. This procedure was repeated for the truncated PSG variants.

\section{Photon Distribution Analysis}

PDA analysis accounts for shot noise, background signal contributions, and binning effects to construct accurate fluorescence parameter histograms ${ }^{31,48}$. PDA analysis was performed using the Seidel Software Tatiana 4.8 software. For this analysis, burstwise photon data was re-binned into time windows of varying lengths: $1 \mathrm{~ms}, 2 \mathrm{~ms}$, and $3 \mathrm{~ms}$. Shorter time windows corresponding to the ends of bursts partway through a time window were discarded. Burstwise FRET efficiency was recalculated from this re-binned data. The resulting histograms were fit globally for each FRET variant to ensure the resulting model is consistent across different time windows sizes. The model used included two static populations corresponding to the limiting state distances with fixed widths $\left(7 \%\right.$ of $\left.\mathrm{R}_{\mathrm{D}(\mathrm{A})}\right)$ as well as a dynamic population corresponding to exchange between these states. Fixed-width states were included to account for apparently static states corresponding either to conformationally static states or dynamic processes not reflected by changes in the FRET efficiency of any given variant. The reaction rates, $k_{\mathrm{AB}}$ and $k_{\mathrm{BA}}$ as well as the population fractions, $\mathrm{A}_{\mathrm{A}}, \mathrm{A}_{\mathrm{B}}$, and $\mathrm{A}_{\mathrm{Dyn} \text {. were }}$ allowed to vary. This model can be summarized as $\left(A \leftrightarrow A^{\prime}\right) \leftrightarrow\left(B \leftrightarrow B^{\prime}\right)$. The mean relaxation time is given by $T_{R}=$ $\frac{1}{k_{R}}=1 /\left(k_{A B}+k_{B A}\right)$. Correction parameters for PDA FRET efficiency histograms were the same as for multiparameter histograms (Supplementary file 1A \& B).

\section{Filtered Fluorescence Correlation Spectroscopy (fFCS)}

For modeling the fFCS we use the following functions.

Species Auto-Correlation Function:

$$
f_{A C}\left(t_{c}\right)=b+\frac{1}{N_{B}\left(1+\frac{t_{C}}{t_{\text {diff }}}\right) \sqrt{1+\frac{t_{C} z_{0}^{2}}{t_{\text {diff }} \omega_{0}^{2}}}}\left(1+\sum_{i}\left(\left|A C_{i}\right| e^{-\frac{t_{C}}{t_{R_{i}}}}-\left|A C_{i}\right|\right)\right)\left(1-\left|A_{\mathrm{Tl}}\right|+\left|A_{\mathrm{Tl}}\right| e^{-\frac{t_{c}}{t_{L}}}\right)
$$

Species Cross-Correlation Function:

$$
f_{C C}\left(t_{c}\right)=b+\frac{1}{N_{B}\left(1+\frac{t_{C}}{t_{d i f f}}\right) \sqrt{1+\frac{t_{C} z_{0}^{2}}{t_{d i f f} \omega_{0}^{2}}}}\left(1-C \sum_{i}\left|C C_{i}\right| e^{-\frac{t_{C}}{t_{R_{i}}}}\right)\left(1-\left|A_{\mathrm{Tl}}\right| e^{-\frac{t_{C}}{t_{L}}}\right)
$$

$$
\sum_{i}\left|C C_{i}\right|=1
$$

Here, $b$ is the baseline value for the correlation function, corresponding to no correlation, $N_{B}$ is the average number of bright molecules in the confocal volume, $t_{c}$ is the correlation time, $t_{\text {diff }}$ is the average diffusion time through the confocal volume, $s=z_{0} / \omega_{0}$ is a geometric factor of the confocal volume as the ratio of widths parallel and perpendicular to the light path of the excitation laser through the sample, $A C_{i}$ and $C C_{i}$ are auto-correlation and crosscorrelation decay amplitudes, $t_{R_{i}}$ are the associated decay times, $C$ is a normalization factor for each individual crosscorrelation curve, and $A_{\mathrm{Tl}}$ and $t_{L}$ are additional decay components to account for long-timescale processes not associated with dynamics that occur below the diffusion time.

\section{Structure generation and system setup}

The PDZ3 and SH3-GuK domains of the PSG core were reconstructed using homology modeling of I-TASSER (Iterative Threading ASSEmbly Refinement) hierarchical approach using multiple threading alignments and iterative 
structural assembly simulations. The I-TASSER method involves four consecutive stages: (a) template identification or threading by LOMETS, (b) structure assembly by replica-exchange Monte Carlo simulations, (c) model selection and structure refinement using REMO and FG-MD, and (d) structure-based function annotation using COFACTOR ${ }^{49}{ }^{50}$. The individual PDZ3, SH3-GuK domains were positioned randomly and our in-house loop reconstruction program 'medusaloop' was utilized to connect the individual domains via flexible linkers to reconstruct the PSG core (Figure 4 - figure supplement 1). The backbone of the GuK domain was kept static while the SH3 and PDZ3 domains were kept flexible to allow adequate sampling of the conformational landscape. Gō constraint was imposed on the intra-PDZ3 (residues 308405), intra-SH3 (residues 431-531) domains and the $\beta$ strand F of GuK (residues 710-724) that folds back to form an antiparallel $\beta$ sheet with $\beta$ strand $\mathrm{E}$ of the SH3 domain. The implementation of Gō-like constraints, based on inter-residue contacts, can provide a reliable model consistent with the experimentally-established native structure of PDZ3 and SH3GuK.

\section{2}

\section{Replica exchange DMD simulations}

Replica exchange DMD (rxDMD) simulations were performed with 18 neighboring replicas in the temperature range of 275-360 K to efficiently sample the conformational free energy landscape. In the rxDMD scheme, temperature was exchanged between replicas at periodic time intervals, according to the Metropolis criterion, to overcome any local energy barriers that may limit efficient sampling of the conformational states. Production runs followed 3000 timestep energy minimization runs, where a DMD timestep corresponded to $\sim 50 \mathrm{fs}$. The conditions for replica exchange were checked every 1000-time units and the frames were saved every 100-time units. Anderson's thermostat was used to maintain temperature at $300 \mathrm{~K}$ in all simulations and the heat exchange factor was set to 0.1 . Each replica simulation lasted for 660 ns that resulted in a total simulation time of $\sim 11.9 \mu$ s.

The thermodynamics of the PSG core was computed using the Weighted Histogram Analysis Method (WHAM) ${ }^{51,52}$. The time evolution of the $R_{g}$ for the 18 simulated replicas is provided in Figure 4 - figure supplement 2 . We observed dynamic, spontaneous conformational exchange in each of the replicas (Figure 4 - figure supplement 2), highlighting the efficient sampling from exchanging temperature between replicate simulations. The 2D free energy landscape was computed as a function of the interdomain distance between PDZ3-SH3 ${ }_{\mathrm{Hook}}$, $\mathrm{PDZ3}-\mathrm{SH} 3_{\text {barrel }}$ and PDZ3-GuK domains. Using the last $400 \mathrm{~ns}$ of replica exchange trajectories, the density of states $g(E)$ was calculated by combining energy histograms from all 18 replicas. The 2D potential of mean force (PMF) as a function of interdomain distance was computed as:

$$
p(R)_{\mathrm{PMF}}=\exp \left(-E_{\mathrm{PMF}} / k_{\mathrm{B}} T\right)
$$

where $E_{\mathrm{PMF}}$ is the free energy integrated over the interdomain distance, $R . T$ is the temperature of interest and $k_{\mathrm{B}}$ is the Boltzmann constant. The free energy contours are scaled in units of $\mathrm{kcal} / \mathrm{mol}$.

The hierarchical clustering program (www.compbio.dundee.ac.uk/downloads/oc) was utilized to group similar conformations of the PSG core. Depending on the pair-wise root-mean square distance (RMSD) matrix, the clustering algorithm iteratively combines nearby clusters. The "cluster distance" was determined based on all pairwise distances between elements of corresponding clusters. We used the mean of all values to compute the distance between two clusters, and the centroid structure of each cluster was chosen with the smallest average distance to other elements in the cluster.

The disulfide bond modeling of the interdomain contacts in PSG was performed for the two basins ( $\alpha$ and $\beta$ ) in the free energy landscape. From the structural ensembles corresponding to the reaction coordinates in the residual contact frequency map, we ranked contact residue pairs and selected the top three pairs near the interface that did not involve the hydrophobic and salt-bridge interactions. The selected pairs were situated at the peripheral sites of the interdomain interface, and the corresponding residues were either polar or weakly hydrophobic in nature ${ }^{34}$.

\section{Rigid Body Docking and Screening of DMD Structures}


Rigid body docking and screening against DMD simulations were performed using the FRET Positioning and Screening (FPS) software ${ }^{27}$. Input distances and uncertainties were taken from seTCSPC in Supplementary file 1E. Initial structure was generated from the representative DMD structure used throughout the main text by removing residues 399428 corresponding to the PDZ3-SH3 linker such that PDZ3 and SH3-GuK are treated as separate rigid bodies. In simulations, PDZ3 is held static while SH3-GuK undergoes a biased random walk with FRET distance restraints imparting forces to guide the docking toward those structures satisfying the restraints. Distances are calculated for rigid bodies by sampling fluorophore accessible volumes (AV) simulated for each fluorophore labeling position. 11000 independent runs were performed from the initial structure for each limiting state distance set from global seTCSPC fitting. All resulting structures for these runs were combined into one dataset and best structure selection was performed from this combined dataset. Representative structures are identified based on minimization of $\chi_{\mathrm{r} \text {,Docking calculated }}^{2}$ between simulated distances for each structure and experimental distances. This procedure was repeated for the distances from the truncated variants. For contact state visualization, an additional restraint per successful DS pair was included for the structure with the shortest distances corresponding to each DS pair, set to $2 \AA+/-2 \AA$.

Screening and FRET robustness analysis were performed in the same software by loading 20871 structures from DMD simulations, simulating AVs for each labeling site in every structure, calculating inter-dye distances by random sampling of the AVs, and again using distances and uncertainties from seTCSPC for comparison. These structures were not modified and therefore included all of residues 308-724. Best representatives were determined for screening in the

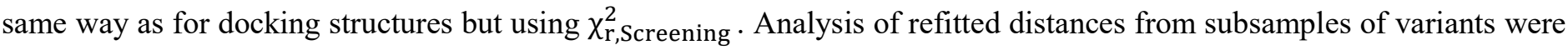
analyzed using the same AV data as for screening. For each structure, interdye distances were calculated from dye AVs and structures were classified based on whether the average percent error of simulated distances for that structure relative to the distances in Supplementary file 4B were below the thresholds corresponding to either basin A, basin B, or neither. Surfaces were calculated corresponding to the PDZ3 center-of-mass positions for all structures classified into either basin, shown in Figure 6. Contours representing classifications of structures are shown in Figure 6 - figure supplement 1. Resulting structures and surfaces from these analyses were visualized in the PyMOL Molecular Graphics System, Version 2.0 (Schrödinger, LLC). Attachment sites and parameters for AV simulations for both docking and screening can be found in Supplementary file 4A.

\section{Disulfide Mapping in PSD-95}

All samples were pre-reduced with $5 \mathrm{mM}$ DTT for one hour followed by desalting using a PD10 column (GE Healthcare) into $20 \mathrm{mM}$ tris $\mathrm{pH} 7.4,150 \mathrm{mM} \mathrm{NaCl}$. Disulfide oxidation reactions were performed at $25^{\circ} \mathrm{C}$ with $2 \mu \mathrm{M}$ protein concentration for the PSG fragment or $0.5 \mu \mathrm{M}$ for full-length PSD-95. Disulfide formation was initiated by the addition of $0.5 \mathrm{mM} \mathrm{CuSO}_{4}$ and $1.75 \mathrm{mM} \mathrm{1,10-phenanthroline.} \mathrm{Time} \mathrm{points} \mathrm{were} \mathrm{quenched} \mathrm{by} \mathrm{adding} 40 \mathrm{mM} \mathrm{N}-$ ethylmaleimide and $10 \mathrm{mM}$ EDTA. Samples were boiled in non-reducing Laemmli sample buffer and run on $10 \%$ or $5 \%$ SDS-PAGE for PSG and full-length, respectively. All experiments were carried out in triplicate. Intensities for native and shifted bands were measured in ImageJ. Percentages of disulfide formation were calculated for each time point and corrected for the presence of higher order oligomers. Each reaction was well fit to a single exponential function to obtain the initial and final extent of disulfide formation along with the reaction rate for each mutant. Replicates were analyzed separately to obtain the average and standard error of measurement (SEM) as well as to estimate the error in the fitted parameters.

\section{Neuroligin Peptide Binding}

Binding experiments used protein constructs described above with a 10-residue synthetic peptide corresponding to the C-terminal 10 residues from rat neuroligin 1a (HPHSHSTTRV), which was synthesized with an N-terminal fluorescein label (5-FAM, GenScript USA Inc. Piscataway, NJ). Fluorescence polarization measurements were carried out in black 96-well plates measured on a Wallac Victor 2 Plate Reader (Perkin Elmer). For all measurements peptide was held at

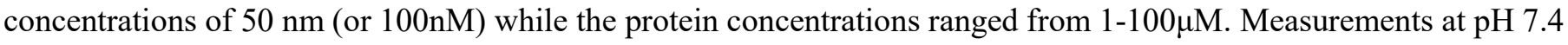


1 were made in $20 \mathrm{mM}$ Tris, $150 \mathrm{mM} \mathrm{NaCl}, 1 \mathrm{mM}$ DTT and $1 \mathrm{mM}$ EDTA. Measurements at $\mathrm{pH}$ 6.0, were made in $20 \mathrm{mM}$

2 MES, $150 \mathrm{mM} \mathrm{NaCl}, 1 \mathrm{mM}$ DTT and 1mM EDTA. Four readings were taken for each well, then averaged. All experiments

3 were carried out in triplicate and fitted with the Hill Equation for a single site binding site model.

4

5 Code Availability

6 MFD is made available at http://www.mpc.hhu.de/en/software. DMD simulation engine is available at http://www.

7 moleculesinaction.com.

8 Acknowledgements

9 This work was supported by NIH (MH081923 to MEB, 1P20GM130451 to HS, and GM119691 to FD), NSF

10 (CAREER MCB 1749778 to HS and CAREER CBET-1553945 to FD); and ERC (AdG2014 hybridFRET (\# 671208) and

11 HHU Connect to GH, HS, and CS. We thank Markus Seeliger (NIH S10OD028478) and Aziz Rangwala for technical

12 assistance with peptide binding experiments.

\section{Author Contributions}

GH, JK, SB and CZ measured and analyzed FRET samples. FSW performed peptide binding experiments. FD and NS created the PSG construct for the DMD simulations. NS performed and analyzed the DMD simulations. FSW, YH and FW performed and analyzed DS mapping experiments. FW designed expression constructs and prepared protein samples. CS and HS supervised confocal FRET experiments. MEB, HS, FD, NS, GH, and SB wrote the paper.

\section{Competing Financial Interests}

The authors declare no conflicts of interest. 


\section{REFERENCES}

1. Han, J.H., Batey, S., Nickson, A.A., Teichmann, S.A. \& Clarke, J. The folding and evolution of multidomain proteins. Nat Rev Mol Cell Biol 8, 319-30 (2007).

2. Ekman, D., Bjorklund, A.K., Frey-Skott, J. \& Elofsson, A. Multi-domain proteins in the three kingdoms of life: orphan domains and other unassigned regions. J Mol Biol 348, 231-43 (2005).

3. Apic, G., Gough, J. \& Teichmann, S.A. Domain combinations in archaeal, eubacterial and eukaryotic proteomes. J Mol Biol 310, 311-25 (2001).

4. Ye, F., Zeng, M. \& Zhang, M. Mechanisms of MAGUK-mediated cellular junctional complex organization. Curr Opin Struct Biol 48, 6-15 (2018).

5. $\quad$ Funke, L., Dakoji, S. \& Bredt, D.S. Membrane-associated guanylate kinases regulate adhesion and plasticity at cell junctions. Annu Rev Biochem 74, 219-45 (2005).

6. Won, S., Levy, J.M., Nicoll, R.A. \& Roche, K.W. MAGUKs: multifaceted synaptic organizers. Curr Opin Neurobiol 43, 94-101 (2017).

7. Ye, F. \& Zhang, M. Structures and target recognition modes of PDZ domains: recurring themes and emerging pictures. Biochem J 455, 1-14 (2013).

8. Saksela, K. \& Permi, P. SH3 domain ligand binding: What's the consensus and where's the specificity? FEBS Lett 586, 2609-14 (2012).

9. Kistner, U., Garner, C.C. \& Linial, M. Nucleotide binding by the synapse associated protein SAP90. FEBS Lett 359, 159-63 (1995).

10. Olsen, O. \& Bredt, D.S. Functional analysis of the nucleotide binding domain of membrane-associated guanylate kinases. J Biol Chem 278, 6873-8 (2003).

11. Kim, E. et al. GKAP, a novel synaptic protein that interacts with the guanylate kinase-like domain of the PSD95/SAP90 family of channel clustering molecules. J Cell Biol 136, 669-78 (1997).

12. McGee, A.W. et al. Structure of the SH3-guanylate kinase module from PSD-95 suggests a mechanism for regulated assembly of MAGUK scaffolding proteins. Mol Cell 8, 1291-301 (2001).

13. Sorensen, C.S. \& Kjaergaard, M. Effective concentrations enforced by intrinsically disordered linkers are governed by polymer physics. Proc Natl Acad Sci U S A 116, 23124-23131 (2019).

14. McCann, J.J., Zheng, L., Chiantia, S. \& Bowen, M.E. Domain orientation in the N-Terminal PDZ tandem from PSD-95 is maintained in the full-length protein. Structure 19, 810-20 (2011).

15. Tompa, P. On the supertertiary structure of proteins. Nat Chem Biol 8, 597-600 (2012).

16. Medina, E., D, R.L. \& Sanabria, H. Unraveling protein's structural dynamics: from configurational dynamics to ensemble switching guides functional mesoscale assemblies. Curr Opin Struct Biol 66, 129-138 (2021).

17. Rademacher, N. et al. Intramolecular domain dynamics regulate synaptic MAGUK protein interactions. Elife 8(2019).

18. Rademacher, N., Kunde, S.A., Kalscheuer, V.M. \& Shoichet, S.A. Synaptic MAGUK multimer formation is mediated by PDZ domains and promoted by ligand binding. Chem Biol 20, 1044-54 (2013).

19. Qian, Y. \& Prehoda, K.E. Interdomain interactions in the tumor suppressor discs large regulate binding to the synaptic protein GukHolder. J Biol Chem 281, 35757-63 (2006).

20. Zhang, J., Lewis, S.M., Kuhlman, B. \& Lee, A.L. Supertertiary structure of the MAGUK core from PSD-95. Structure 21, 402-13 (2013).

21. Korkin, D. et al. Structural modeling of protein interactions by analogy: application to PSD-95. PLoS Comput Biol 2, e153 (2006).

22. McCann, J.J. et al. Supertertiary structure of the synaptic MAGuK scaffold proteins is conserved. Proc Natl Acad Sci US A 109, 15775-80 (2012).

23. Yanez Orozco, I.S. et al. Identifying weak interdomain interactions that stabilize the supertertiary structure of the N-terminal tandem PDZ domains of PSD-95. Nat Commun 9, 3724 (2018).

24. Irie, M. et al. Binding of neuroligins to PSD-95. Science 277, 1511-5 (1997).

25. Fu, Z., Washbourne, P., Ortinski, P. \& Vicini, S. Functional excitatory synapses in HEK293 cells expressing neuroligin and glutamate receptors. $J$ Neurophysiol 90, 3950-7 (2003).

26. Saro, D. et al. A thermodynamic ligand binding study of the third PDZ domain (PDZ3) from the mammalian neuronal protein PSD-95. Biochemistry 46, 6340-52 (2007). 
27. Dimura, M. et al. Quantitative FRET studies and integrative modeling unravel the structure and dynamics of biomolecular systems. Curr Opin Struct Biol 40, 163-185 (2016).

28. Opanasyuk, O. et al. Unraveling multi-state molecular dynamics in single-molecule FRET experiments--Part II: Quantitative analysis of multi-state kinetic networks. arXiv preprint arXiv:2107.14791 (2021).

29. Barth, A. et al. Unraveling multi-state molecular dynamics in single-molecule FRET experiments-Part I: Theory of FRET-Lines. arXiv preprint arXiv:2107.14770 (2021).

30. Tavares, G.A., Panepucci, E.H. \& Brunger, A.T. Structural characterization of the intramolecular interaction between the SH3 and guanylate kinase domains of PSD-95. Mol Cell 8, 1313-25 (2001).

31. Kalinin, S., Felekyan, S., Antonik, M. \& Seidel, C.A. Probability distribution analysis of single-molecule fluorescence anisotropy and resonance energy transfer. J Phys Chem B 111, 10253-62 (2007).

32. Felekyan, S., Kalinin, S., Sanabria, H., Valeri, A. \& Seidel, C.A. Filtered FCS: species auto- and cross-correlation functions highlight binding and dynamics in biomolecules. Chemphyschem 13, 1036-53 (2012).

33. Kurochkina, N. \& Guha, U. SH3 domains: modules of protein-protein interactions. Biophys Rev 5, 29-39 (2013).

34. Bass, R.B., Butler, S.L., Chervitz, S.A., Gloor, S.L. \& Falke, J.J. Use of site-directed cysteine and disulfide chemistry to probe protein structure and dynamics: applications to soluble and transmembrane receptors of bacterial chemotaxis. Methods Enzymol 423, 25-51 (2007).

35. Kalinin, S. et al. A toolkit and benchmark study for FRET-restrained high-precision structural modeling. Nat Methods 9, 1218-25 (2012).

36. Fernandez, E. et al. Targeted tandem affinity purification of PSD-95 recovers core postsynaptic complexes and schizophrenia susceptibility proteins. Mol Syst Biol 5, 269 (2009).

37. McCann, J.J., Choi, U.B. \& Bowen, M.E. Reconstitution of multivalent PDZ domain binding to the scaffold protein PSD-95 reveals ternary-complex specificity of combinatorial inhibition. Structure 22, 1458-66 (2014).

38. Chen, J.W., Romero, P., Uversky, V.N. \& Dunker, A.K. Conservation of intrinsic disorder in protein domains and families: I. A database of conserved predicted disordered regions. J Proteome Res 5, 879-87 (2006).

39. Zeng, M., Ye, F., Xu, J. \& Zhang, M. PDZ Ligand Binding-Induced Conformational Coupling of the PDZ-SH3GK Tandems in PSD-95 Family MAGUKs. J Mol Biol 430, 69-86 (2018).

40. Nomme, J. et al. The Src homology 3 domain is required for junctional adhesion molecule binding to the third PDZ domain of the scaffolding protein ZO-1. J Biol Chem 286, 43352-60 (2011).

41. Laursen, L., Karlsson, E., Gianni, S. \& Jemth, P. Functional interplay between protein domains in a supramodular structure involving the postsynaptic density protein PSD-95. J Biol Chem 295, 1992-2000 (2020).

42. McCann, J.J., Choi, U.B., Zheng, L., Weninger, K. \& Bowen, M.E. Optimizing methods to recover absolute FRET efficiency from immobilized single molecules. Biophys $J$ 99, 961-70 (2010).

43. Kudryavtsev, V. et al. Combining MFD and PIE for accurate single-pair Forster resonance energy transfer measurements. Chemphyschem 13, 1060-78 (2012).

44. Ma, J. et al. High Precision FRET at Single-molecule Level for Biomolecule Structure Determination. J Vis Exp (2017).

45. Maus, M. et al. An experimental comparison of the maximum likelihood estimation and nonlinear least-squares fluorescence lifetime analysis of single molecules. Anal Chem 73, 2078-86 (2001).

46. Peulen, T.O., Opanasyuk, O. \& Seidel, C.A.M. Combining Graphical and Analytical Methods with Molecular Simulations To Analyze Time-Resolved FRET Measurements of Labeled Macromolecules Accurately. $J$ Phys Chem B 121, 8211-8241 (2017).

47. Hellenkamp, B. et al. Precision and accuracy of single-molecule FRET measurements-a multi-laboratory benchmark study. Nat Methods 15, 669-676 (2018).

48. Kalinin, S., Valeri, A., Antonik, M., Felekyan, S. \& Seidel, C.A. Detection of structural dynamics by FRET: a photon distribution and fluorescence lifetime analysis of systems with multiple states. $J$ Phys Chem B 114, 798395 (2010).

49. Roy, A., Xu, D., Poisson, J. \& Zhang, Y. A protocol for computer-based protein structure and function prediction. $J$ Vis Exp, e3259 (2011).

50. Roy, A., Kucukural, A. \& Zhang, Y. I-TASSER: a unified platform for automated protein structure and function prediction. Nat Protoc 5, 725-38 (2010).

51. Feig, M., Karanicolas, J. \& Brooks, C.L., 3rd. MMTSB Tool Set: enhanced sampling and multiscale modeling methods for applications in structural biology. J Mol Graph Model 22, 377-95 (2004). 
52. Kumar, S., Rosenberg, J.M., Bouzida, D., Swendsen, R.H. \& Kollman, P.A. THE weighted histogram analysis method for free-energy calculations on biomolecules. I. The method. Journal of Computational Chemistry 13, 1011-1021 (1992). 


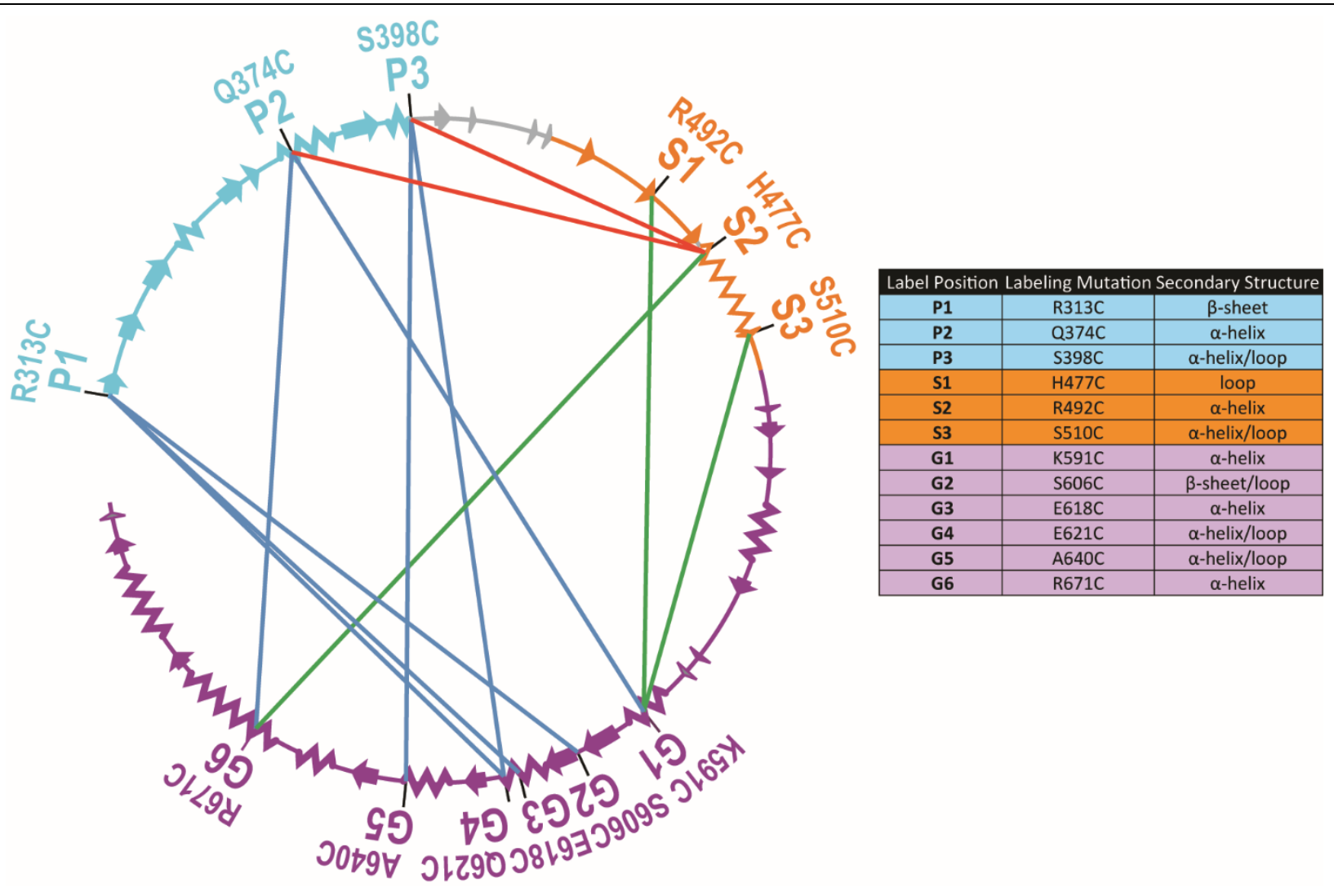

Figure 1-figure supplement 1. Connectivity in the FRET Network and Labeling Site Environments. The primary sequence of PSD-95 is shown in a circular representation with each domain colored: PDZ3 (cyan), SH3 (orange) and GuK (purple). The secondary structural elements are indicated: $\alpha$ helices (zig zag) and $\beta$ sheets (arrows). The position of each labeling site is indicated by the domain and order in the primary sequence. Specific details for each labeling site are also provided in the associated table. The FRET pairs used for measurements are indicated by lines connecting the labeling sites used in that variant with FRET pairs spanning PDZ3-GuK (blue), PDZ3-SH3 (red), and SH3-GuK (green) 


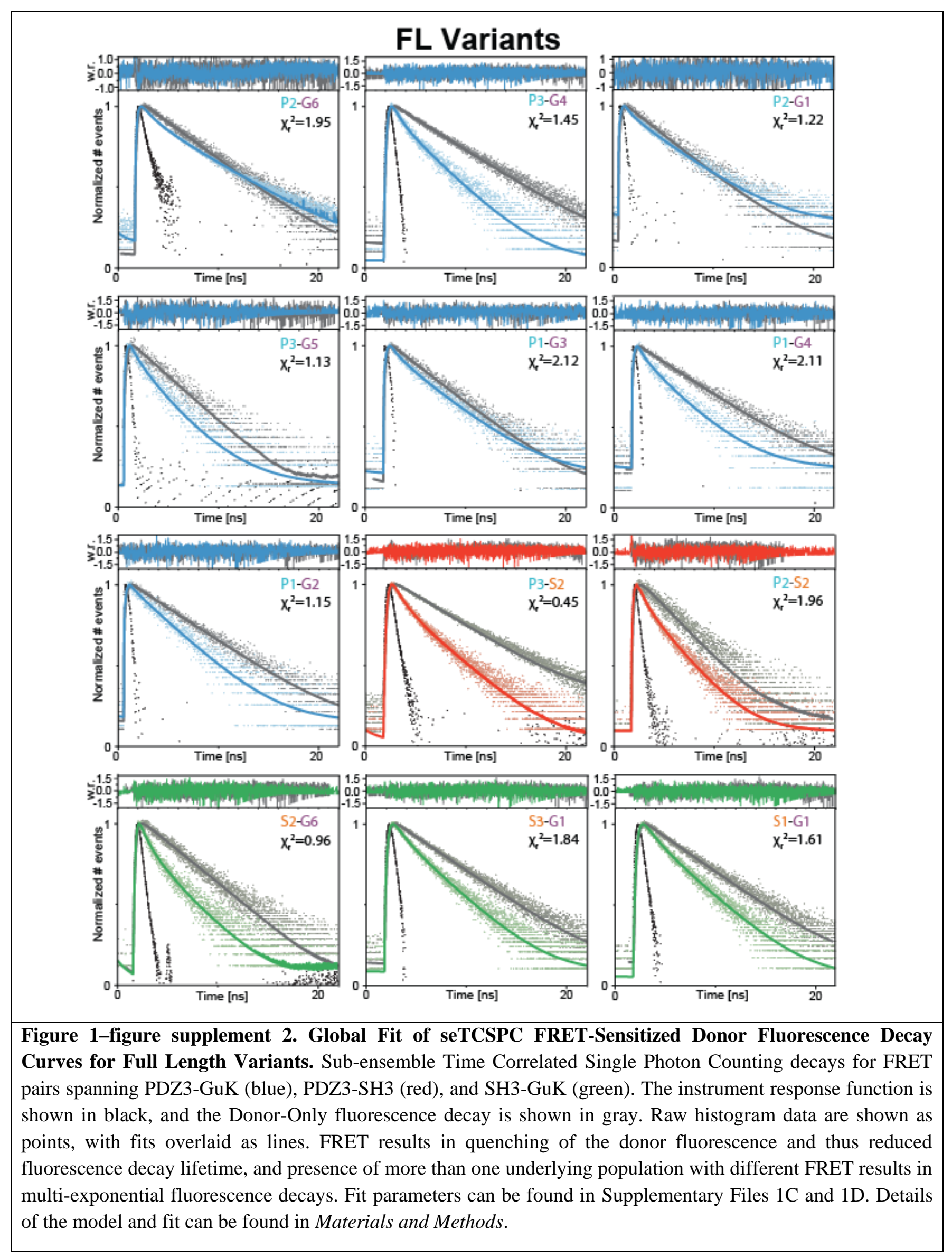




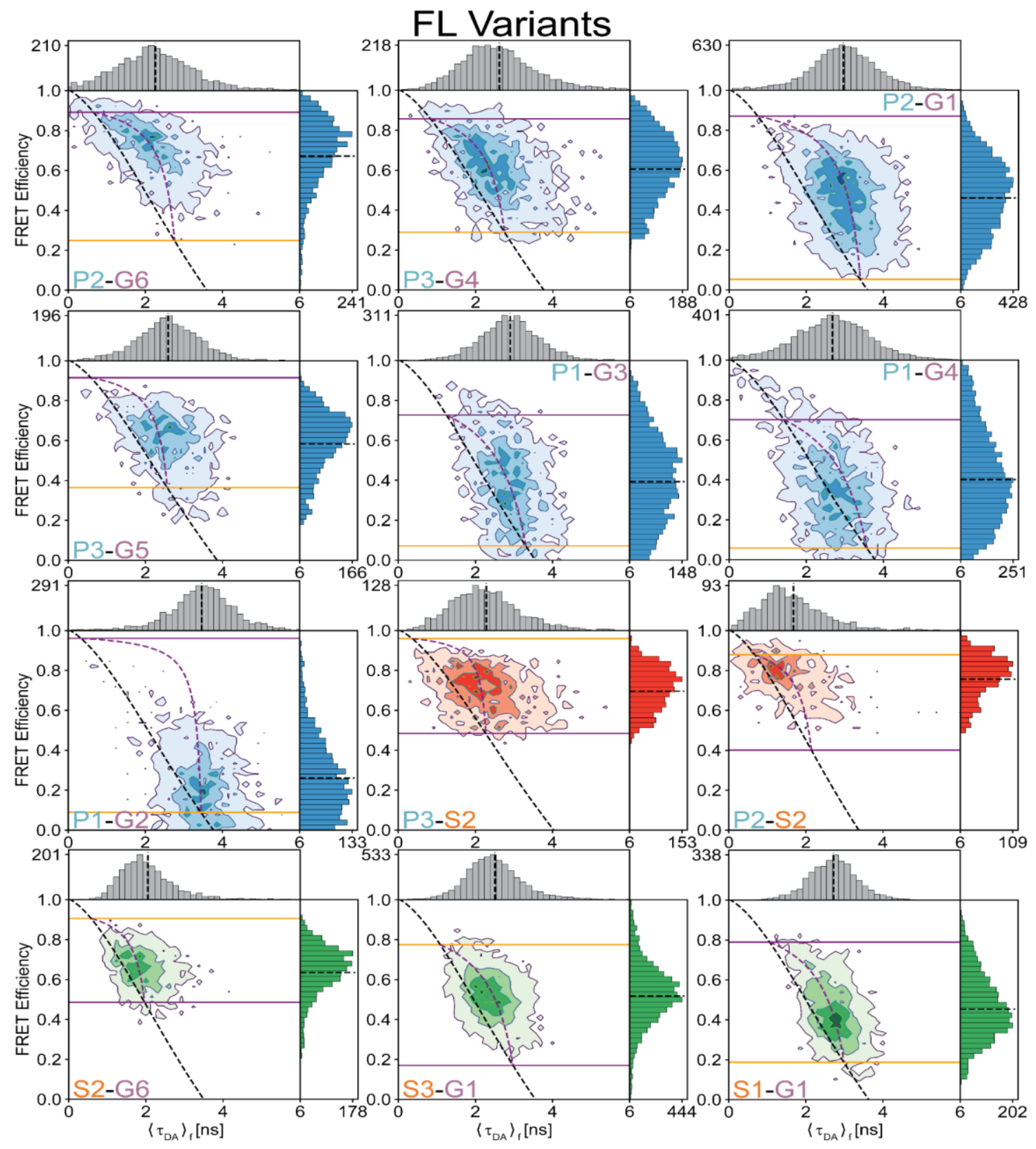

Figure 1-figure supplement 3. Multiparameter Fluorescence Histograms of Full-Length PSD-95 FRET Variants. MFD Histograms for FRET pairs spanning PDZ3-GuK (blue), PDZ3-SH3 (red), and SH3-GuK (green) as noted in each panel. Variant details can be found in Figure S1. Overlaid on the contour plots are the static FRET-lines (black, dashed), dynamic FRET-lines (purple, dashed), and solid horizontal lines corresponding to the limiting states A (orange) and B (purple) from seTCSPC. Also given are the burst-wise average values for the mean donor fluorescence lifetime $\left(\left\langle\left\langle\tau_{D A}\right\rangle_{f}\right\rangle\right)$ and mean FRET Efficiency $(\langle E\rangle)$ (black, dashed lines in 2D histograms). Dynamic exchange is immediately evident from broadening and skew rightward from the static FRET-line for each variant. Correction parameters for these histograms can be found in Supplementary Files 1A and 1B while parameters for the static and dynamic FRET-lines can be found in Supplementary Files 1F and 1G, respectively. 


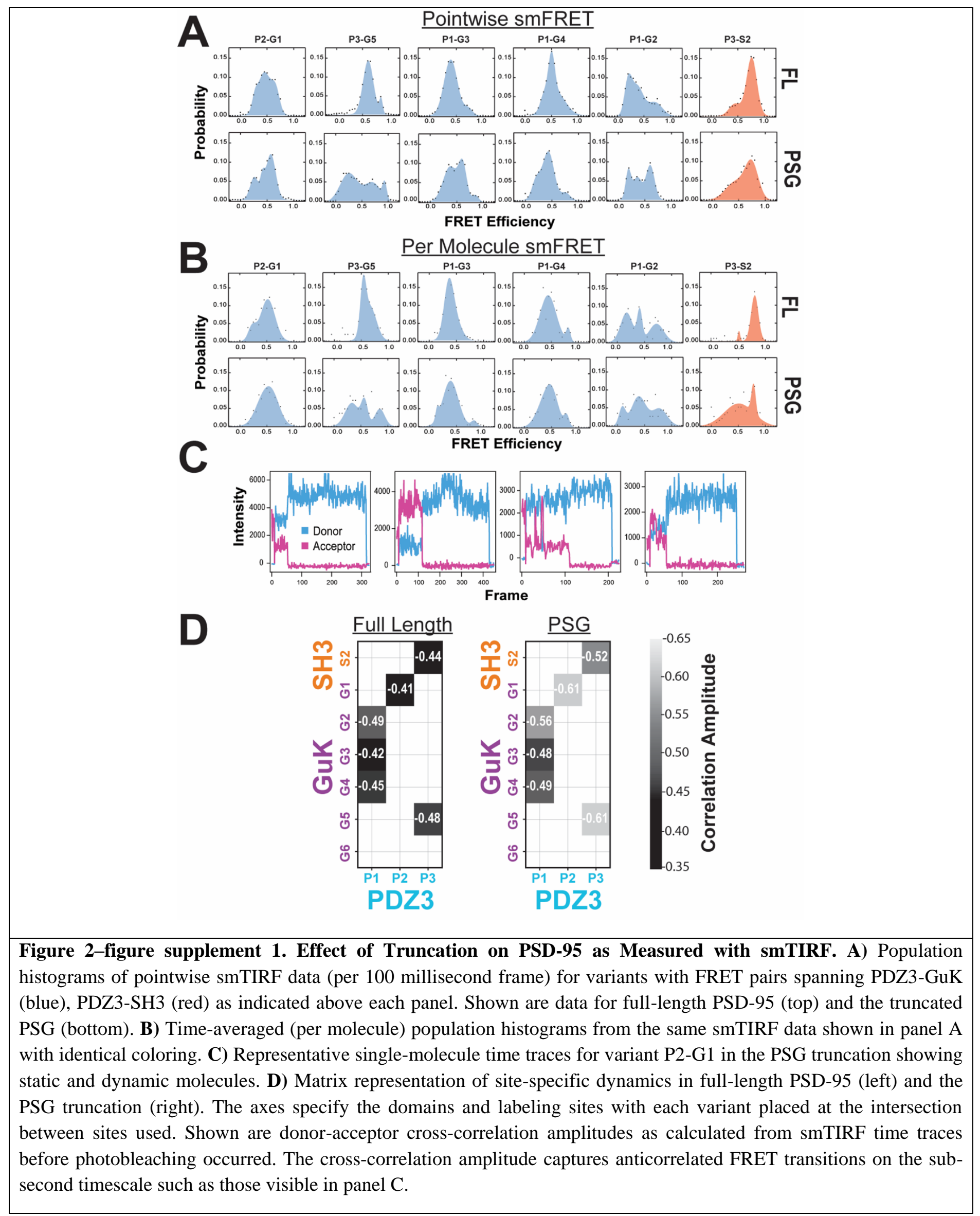




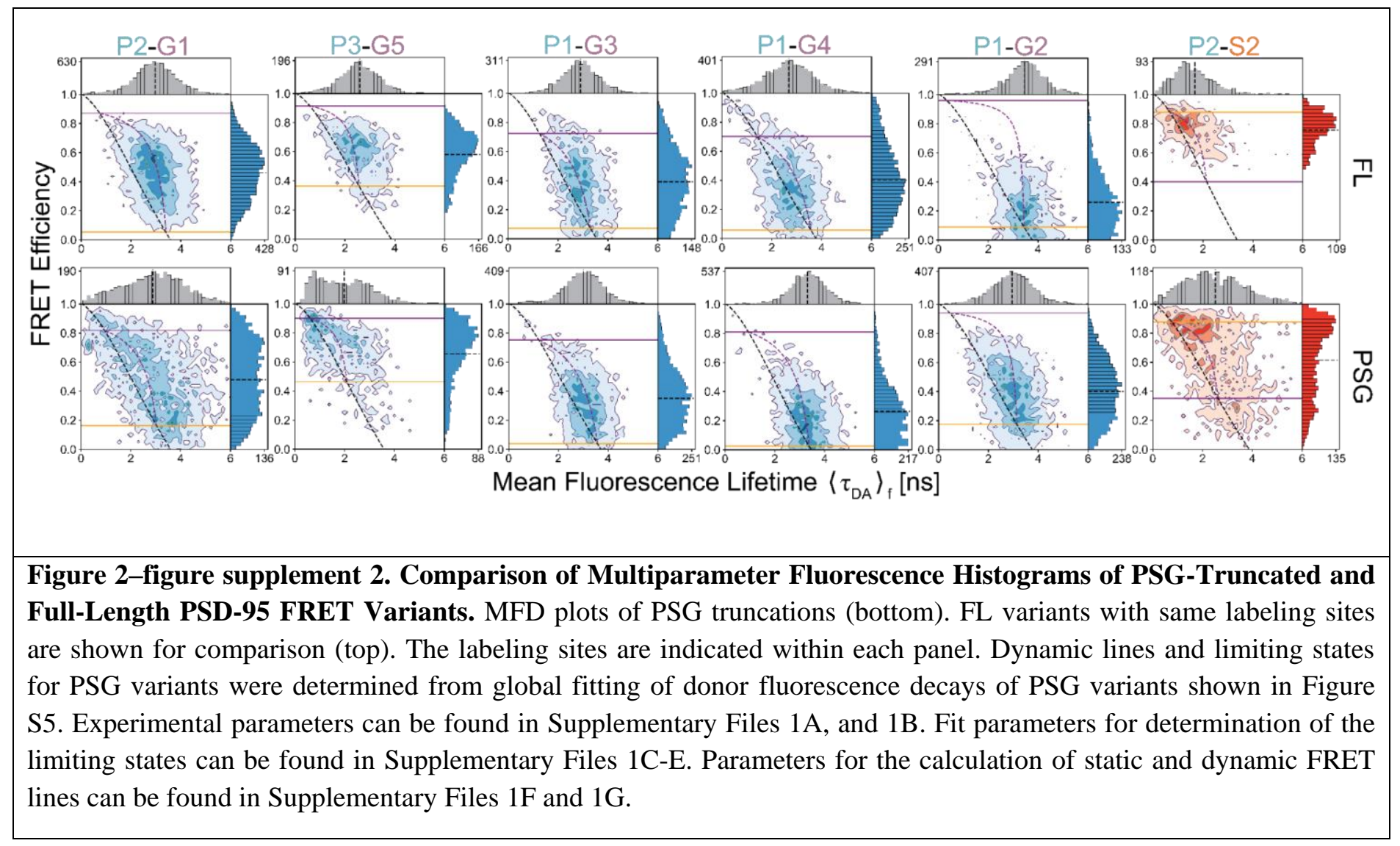




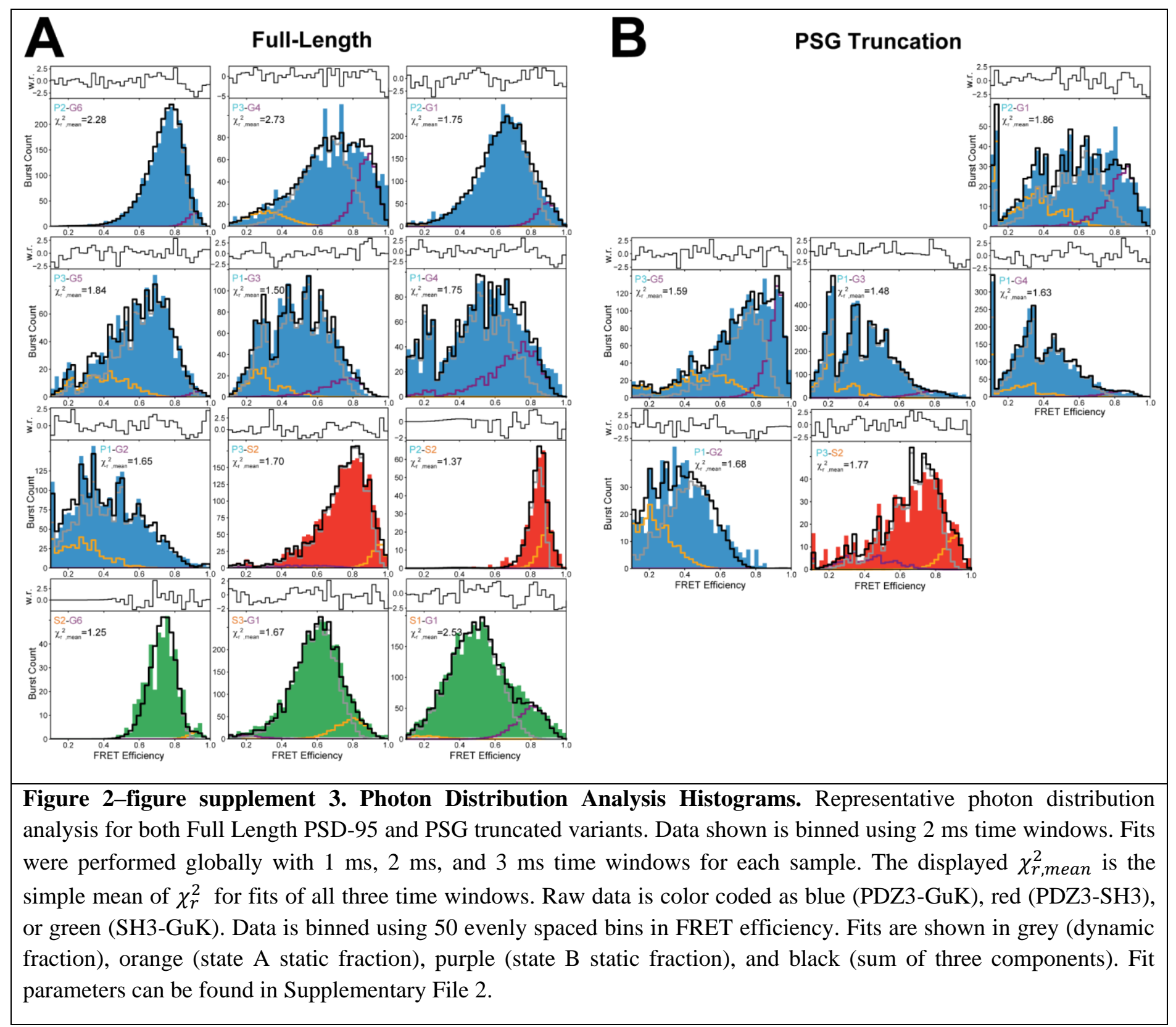




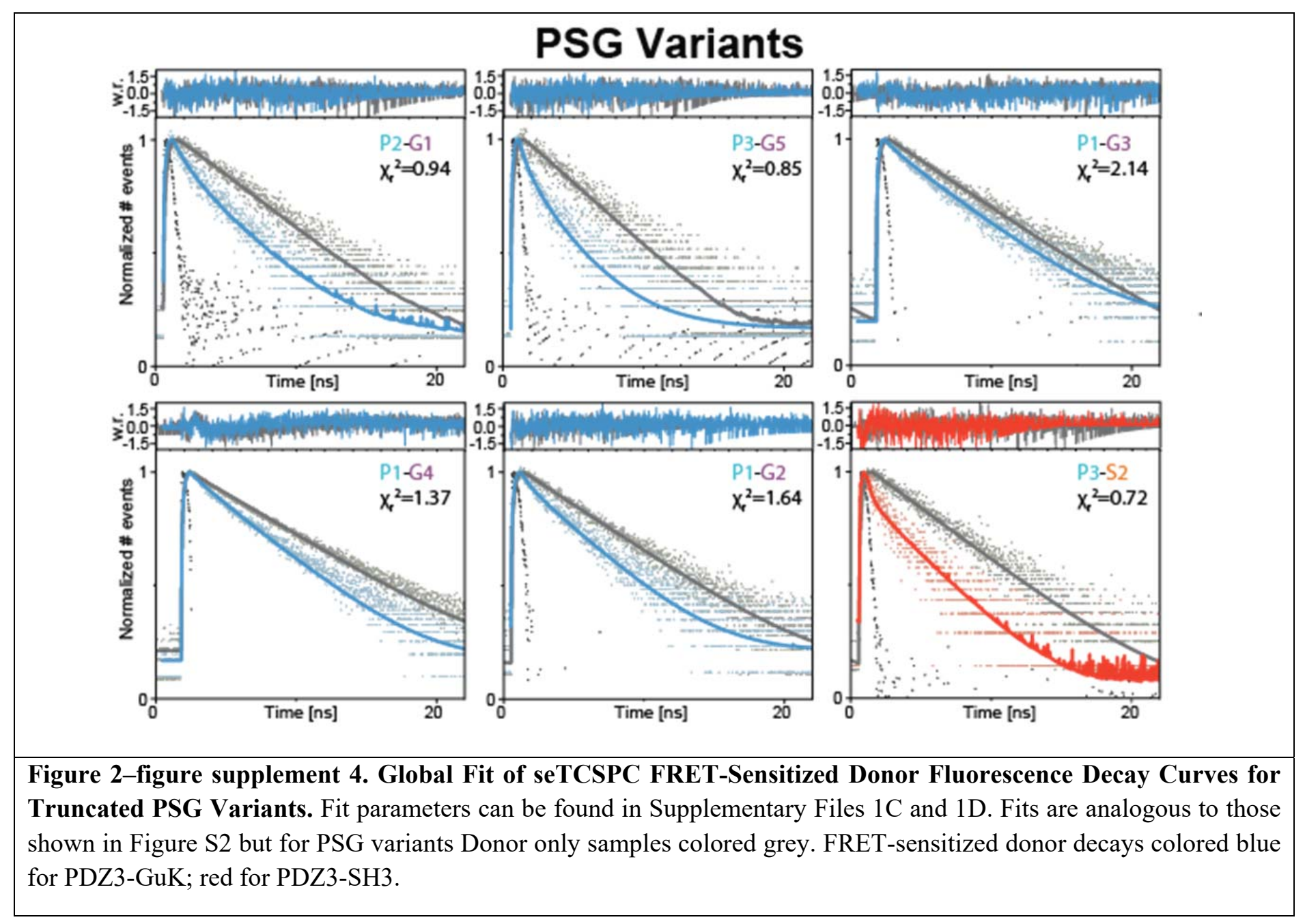




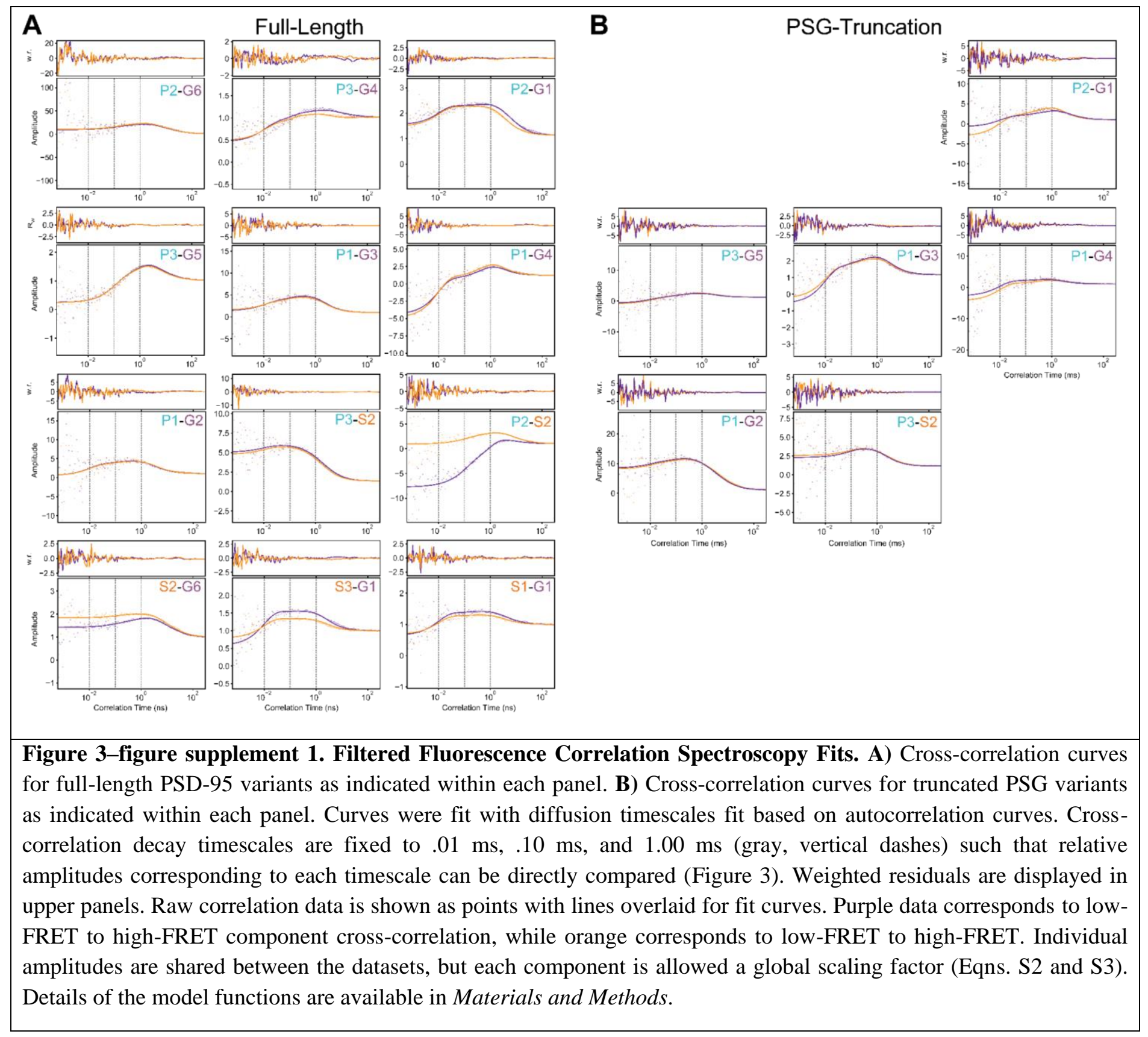




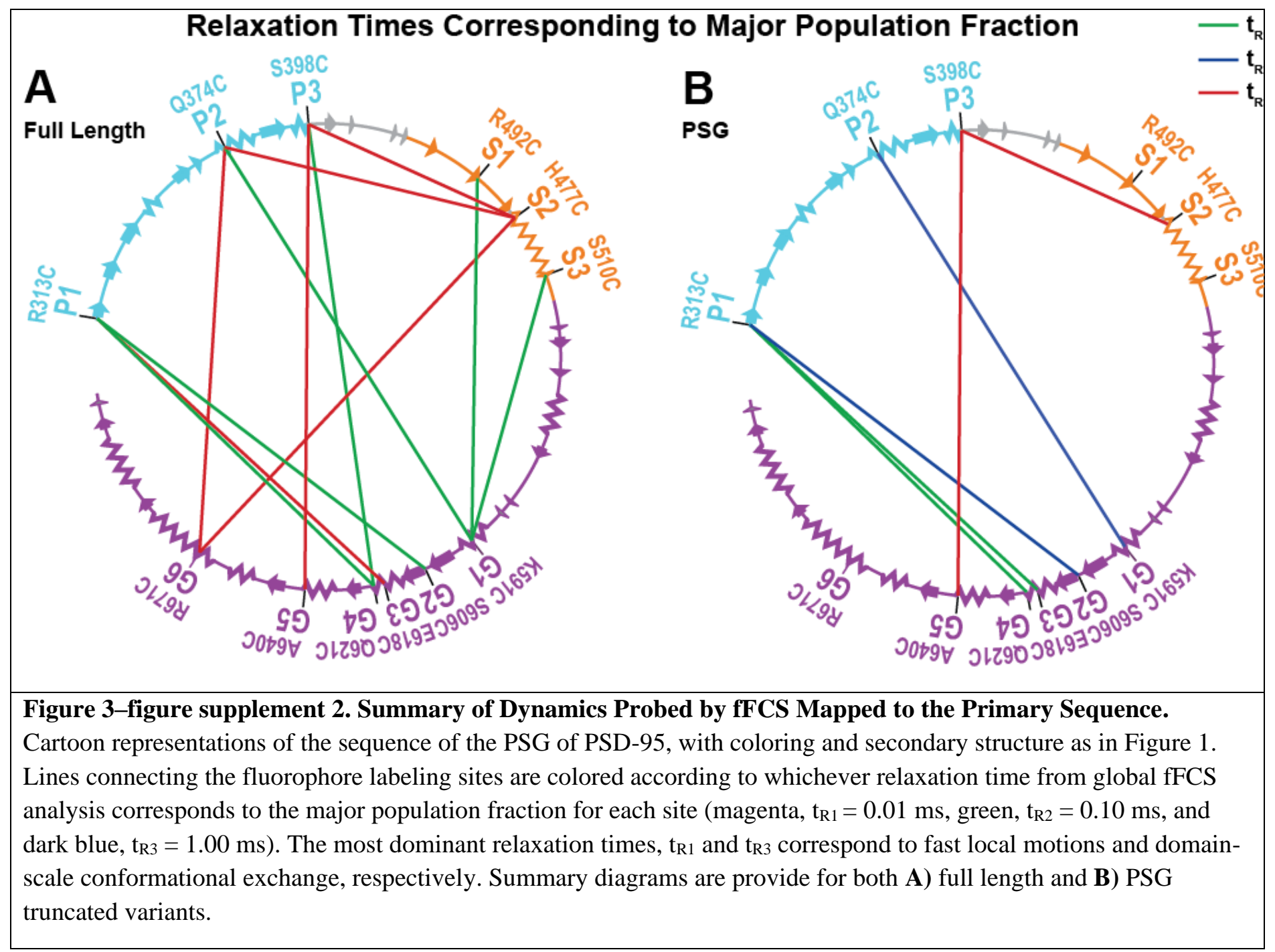




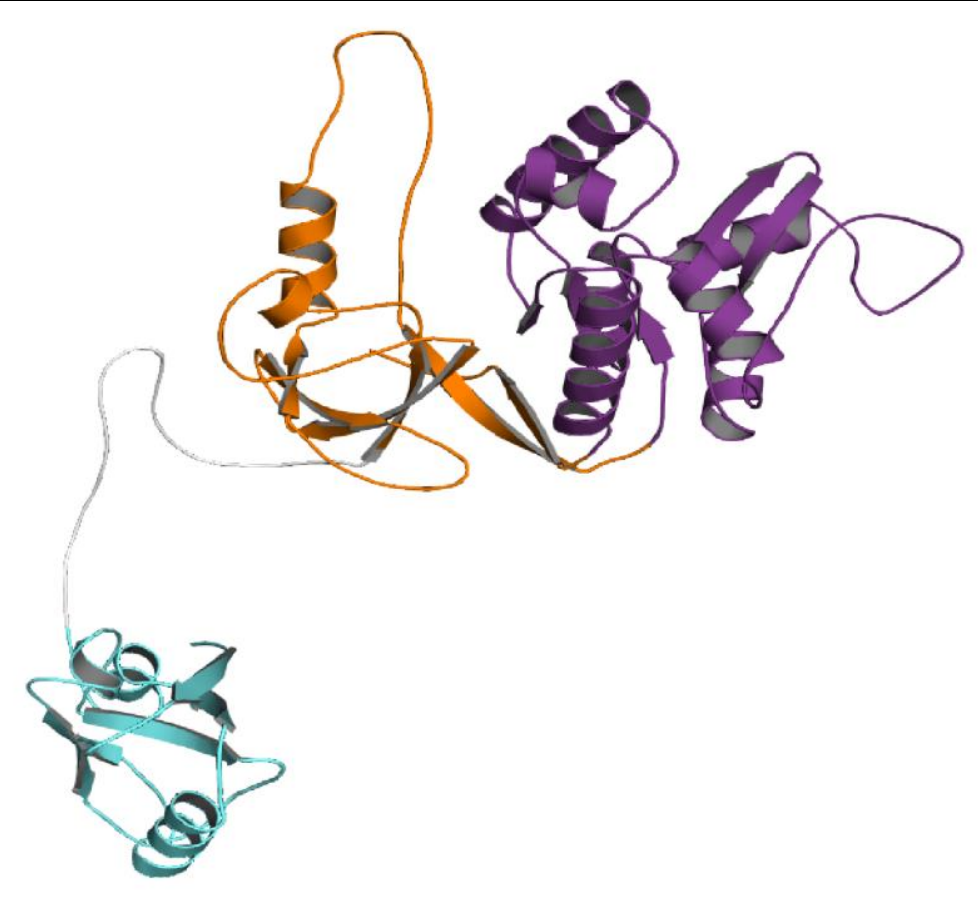

Figure 4-figure supplement 1. The Starting Conformation for the PSG Supramodule from PSD-95 Used in DMD Simulations. Cartoon representation of the initial model for DMD simulations showing PDZ3 (cyan), SH3 (orange) and GuK (purple). To avoid biasing the interactions, PDZ3 was positioned away from the SH3GuK without any contacts. The model was constructed from the crystal structures of PDZ3 (1TP5) and SH3GuK (1KJW). PDZ3 was placed in a random orientation without interdomain contacts to avoid bias. The PDZ3SH3 linker and missing loops were reconstructed using our in-house loop reconstruction program 'medusaloop'. 


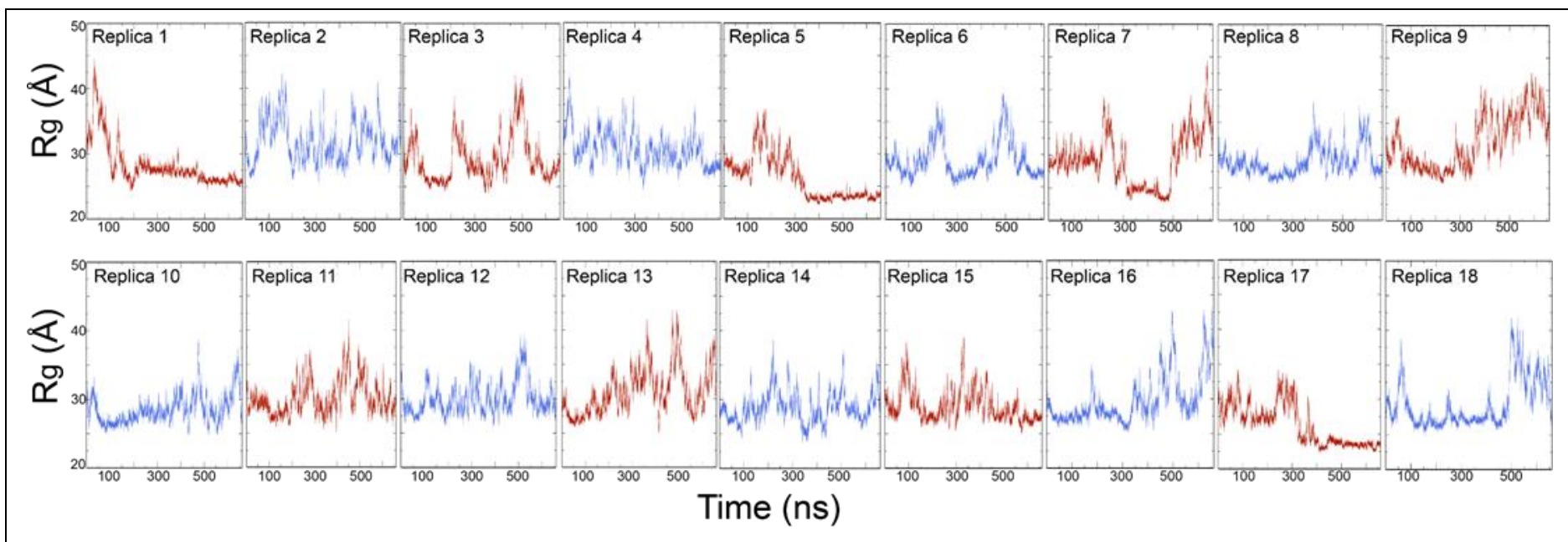

Figure 4-figure supplement 2. Time evolution of the radius of gyration $\left(R_{g}\right)$ of PSG supramodule for 18 replicas DMD simulations. The total simulation time for each replica is $660 \mathrm{~ns}$ and amounts to a total simulation time of 11.9 $\mu \mathrm{s}$. We used the last $400 \mathrm{~ns}$ of replica exchange trajectories for the statistical analysis. 
A Representative conformations of PSG corresponding to a-basin

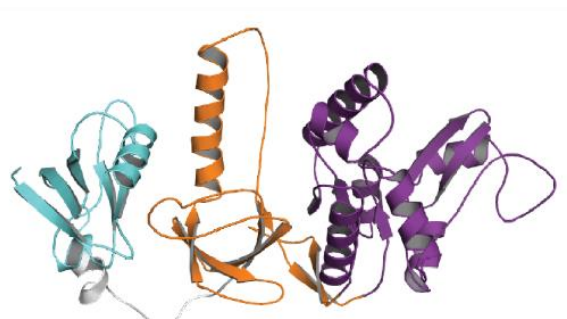

$42.8 \%$

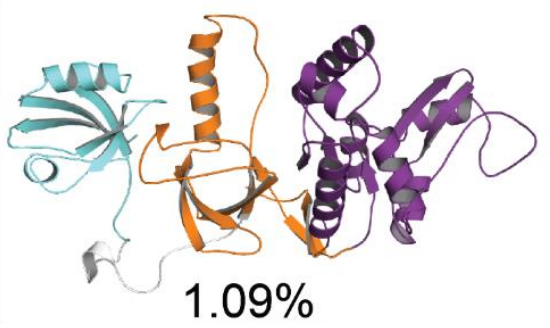

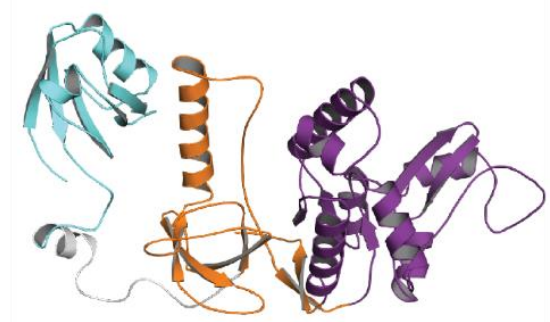

$37.3 \%$

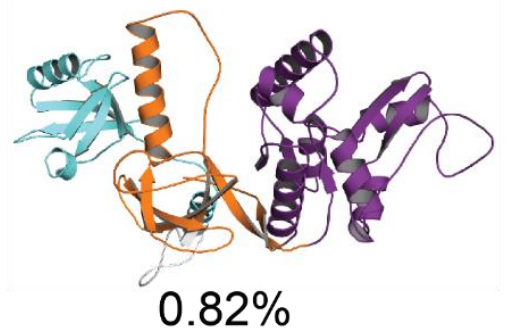

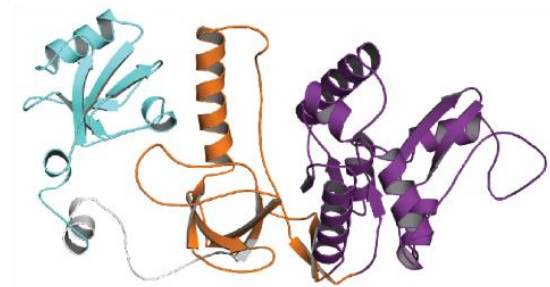

$16.1 \%$

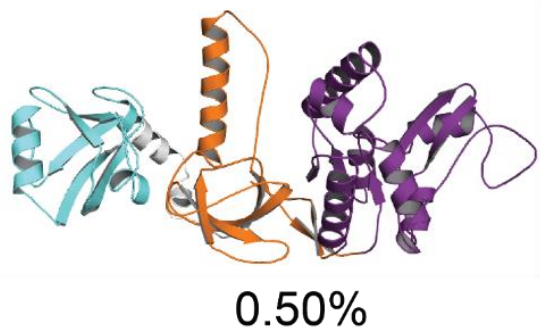

\section{B Representative conformations of PSG corresponding to $\beta$-basin}

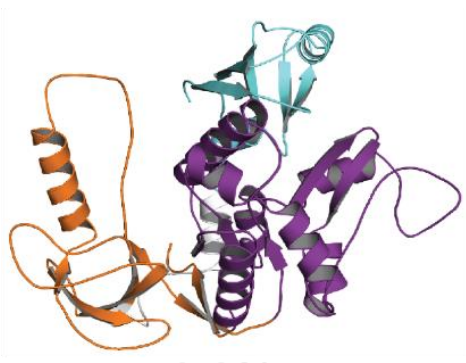

$76.8 \%$
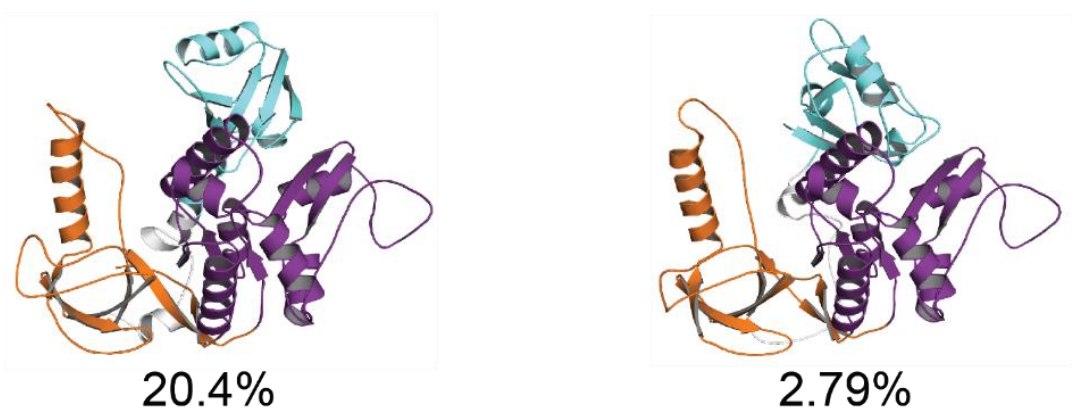

Figure 4-figure supplement 3. Representative Conformations of PSG Supramodule from PSD-95. A) Representative conformations and their population occupancy in the highly sampled $\alpha$-basin with PDZ3 interacting with the SH3 domain. A multiplicity of states is sampled within the fuzzy $\alpha$-basin. The top centroid cluster in the $\alpha$ basin shows PDZ3 to reorient away from the HOOK helix and sample the $\beta 1-\beta 2$ loop (RT loop in canonical SH3 domain). The pairwise contact maps from DMD show that interactions within the $\alpha$-basin involved degenerate electrostatic interactions engaged by all conformations with occasional hydrophobic interactions leading an occluded PDZ3 binding pocket. B) Representative conformations and their population in the $\beta$-basin with PDZ3 interacting with the GuK domain of PSG. The $\beta$-basin was comparatively well defined due to involvement of exposed hydrophobic residues in $\beta 3$ - $\alpha 1$ of PDZ3 and a relatively hydrophobic surface in GuK formed by $\alpha 7, \alpha 5$, and $\beta 10-11$ (e.g., L349F684, L342-L608), which support a limited range of conformations. Both basins infrequently engaged the canonical GLGF motif, which would prevent peptide binding to PDZ3. An RMSD cutoff of $8.0 \AA$ was used to select the centroid clusters of $\alpha$ - and $\beta$-basins. PDZ3 is colored cyan, SH3 colored orange, and GuK colored purple. 


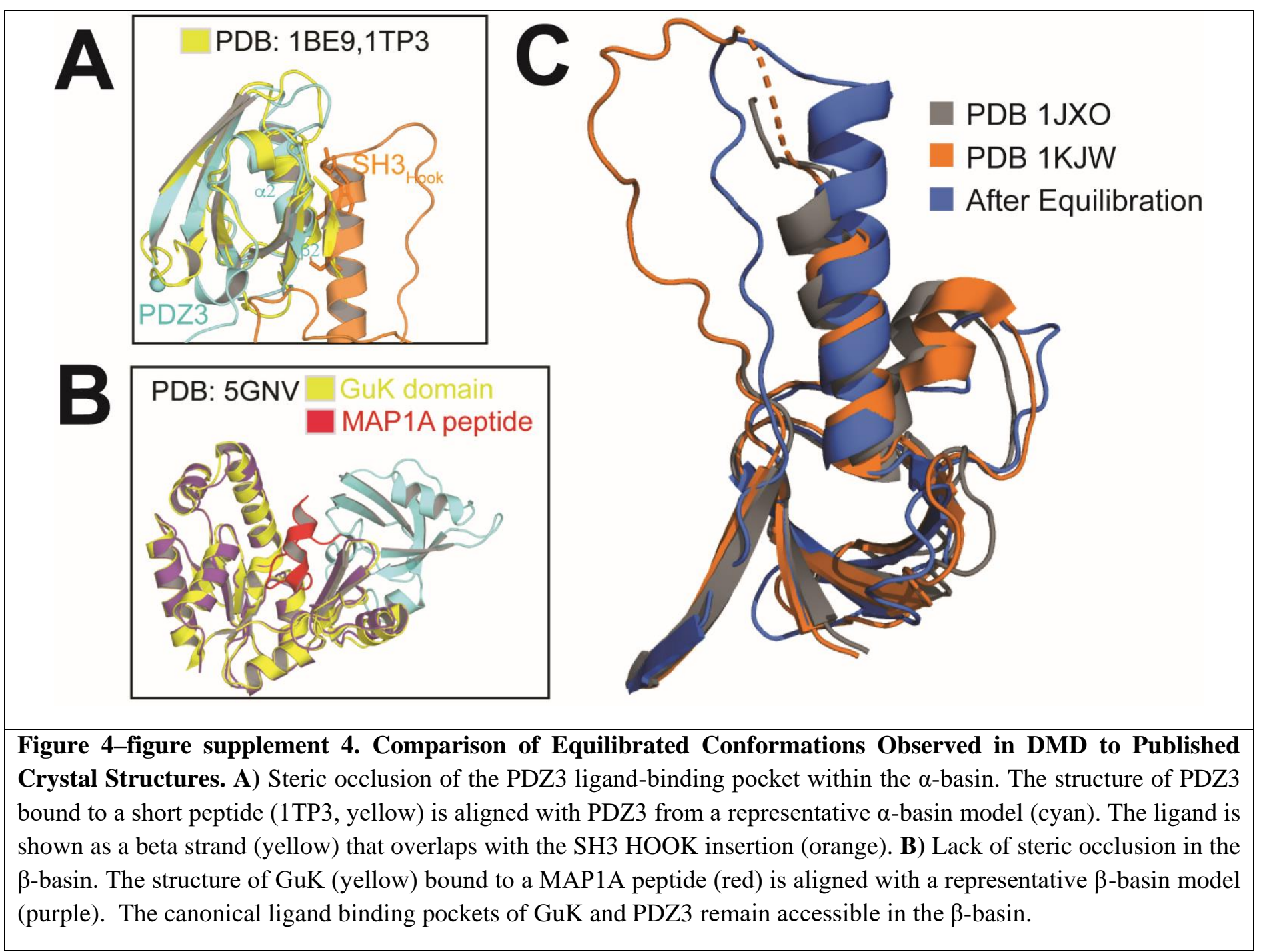




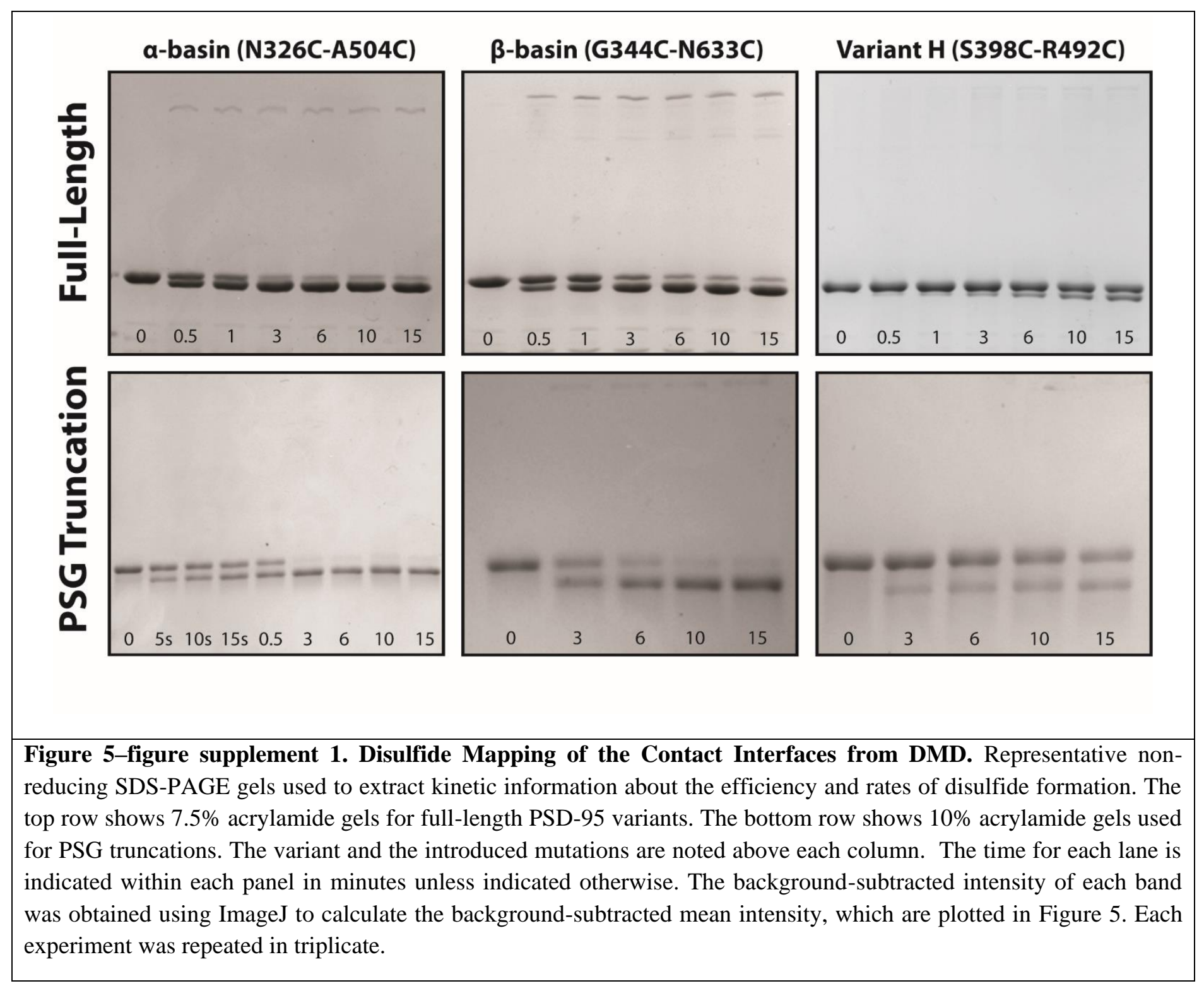




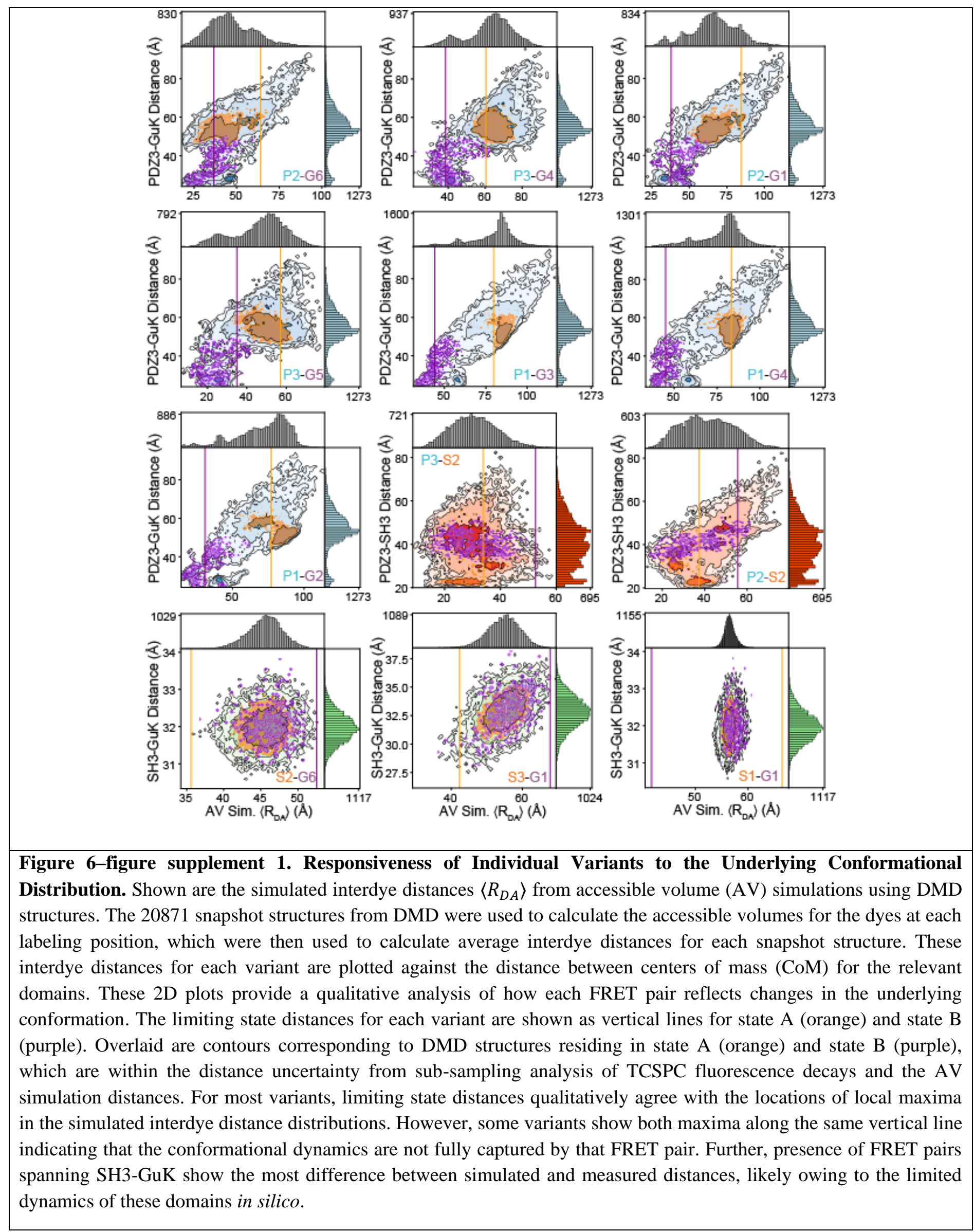




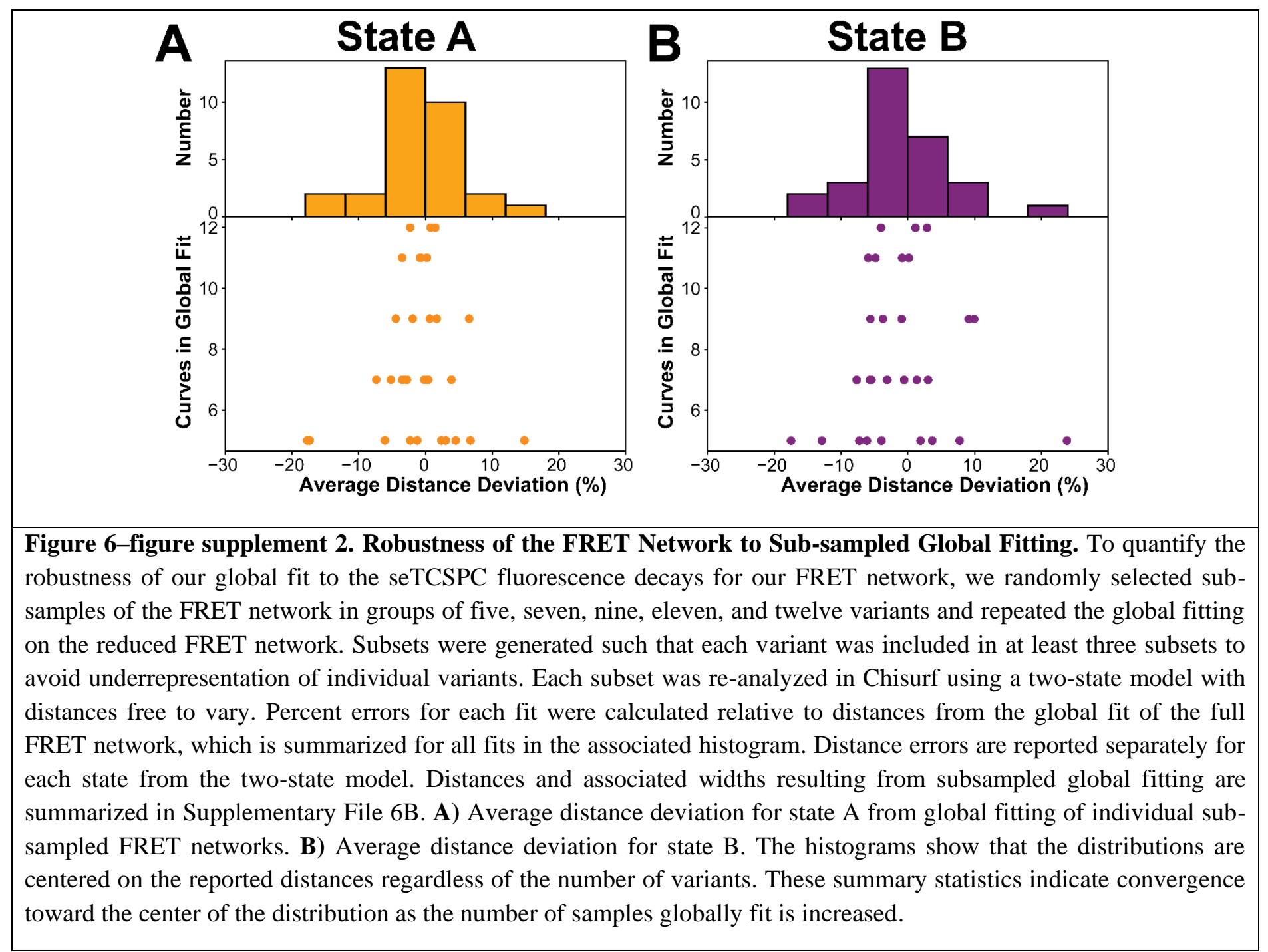




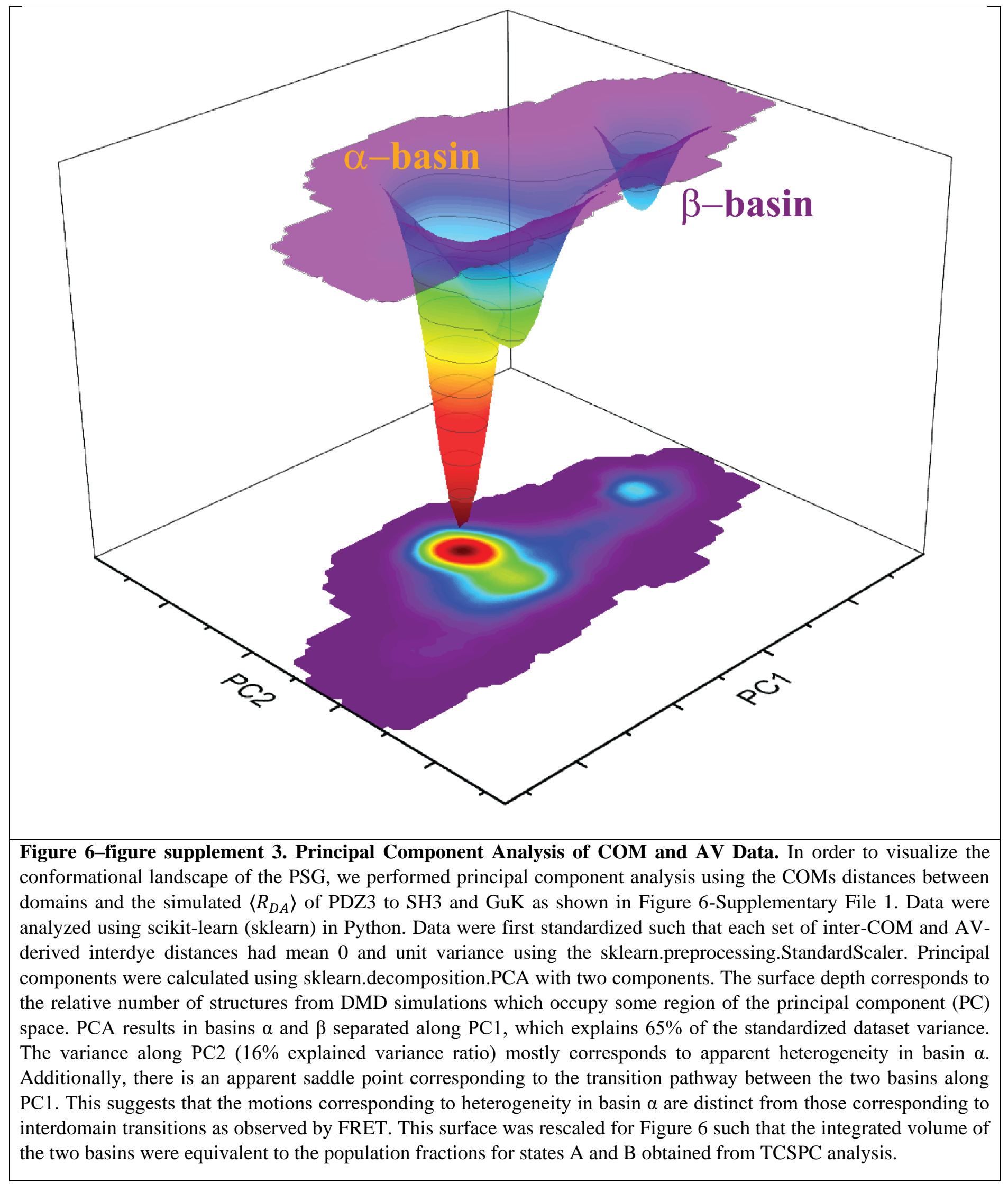




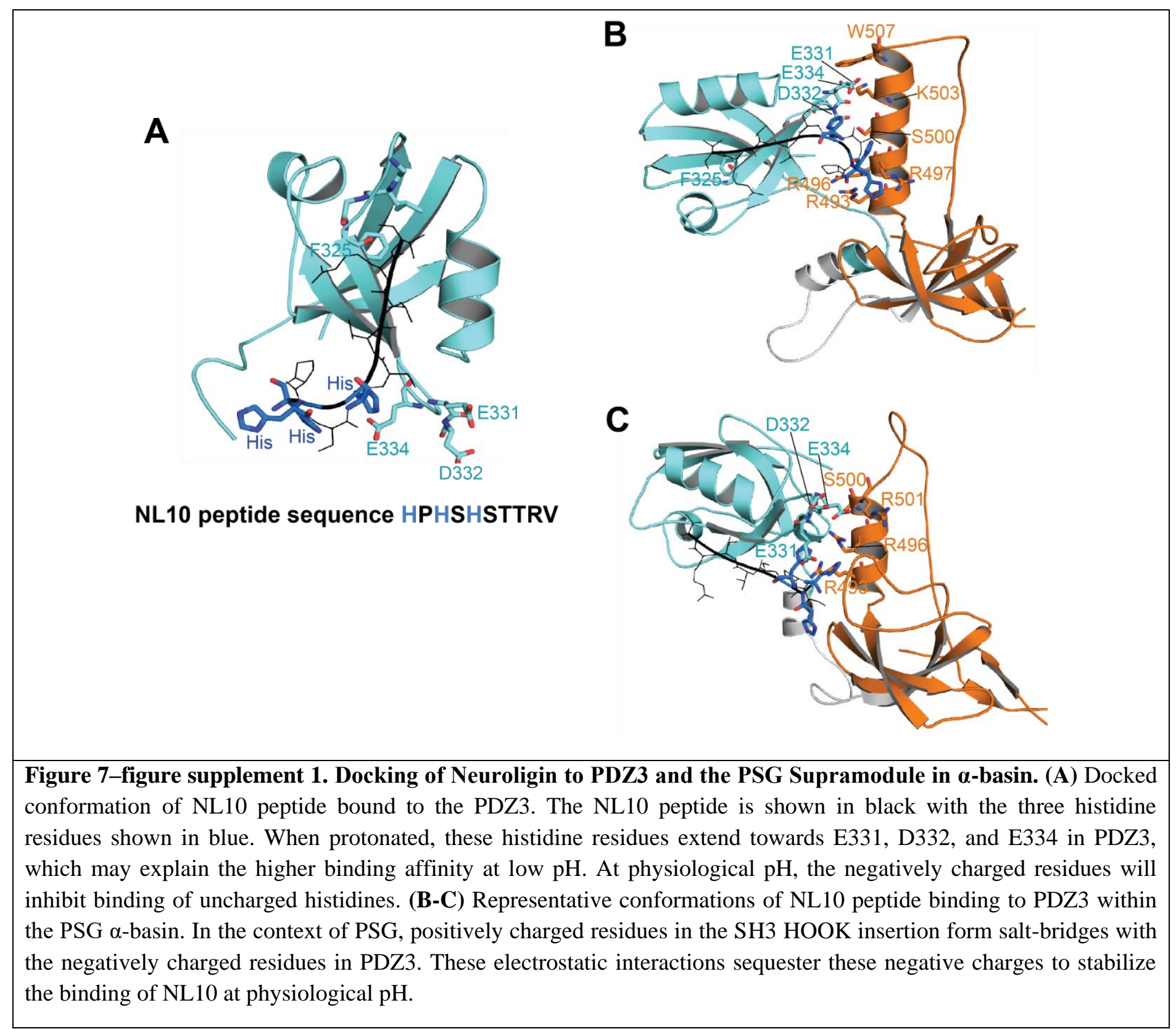

